\title{
FUNCTIONS THAT PRESERVE CERTAIN CLASSES OF SEQUENCES AND LOCALLY LIPSCHITZ FUNCTIONS
}

\author{
Lipsy Gupta and Subiman Kundu \\ Indian Institute of Technology Delhi, Department of Mathematics \\ New Delhi-110016, India; lipsy1247@gmail.com \\ Indian Institute of Technology Delhi, Department of Mathematics \\ New Delhi-110016, India; skundu@maths.iitd.ac.in \\ Dedicated to Professor Gerald Beer
}

\begin{abstract}
The class of cofinally complete metric spaces lies between the class of complete metric spaces and that of compact metric spaces. It is known that a metric space $(X, d)$ is cofinally complete if and only if every real-valued continuous function on $(X, d)$ is cofinally Cauchy regular, where a function is said to be cofinally Cauchy regular or CC-regular for short if it preserves cofinally Cauchy sequences. Recently in 2017, Keremedis has defined almost bounded functions and AUC spaces [22]. We show that an AUC space is nothing but a cofinally complete metric space and an almost bounded function is nothing but a CC-regular function. Also in this paper, we study boundedness of various Lipschitz-type functions which are CC-regular as well and find equivalent characterizations of metric spaces on which such functions are uniformly continuous. Finally we explore some properties of cofinally Bourbaki-Cauchy regular functions, where a function is said to be cofinally Bourbaki-Cauchy regular if it preserves cofinally Bourbaki-Cauchy sequences [17] and find their relation with CC-regular functions.
\end{abstract}

\section{Introduction}

The concepts of completeness and compactness play a very important role in the theory of metric spaces. Some classes of metric spaces satisfying properties stronger than completeness and weaker than compactness have been the subject of study for a number of articles over the decades. One such well-known metric space is Atsuji space, also widely known as UC space. A metric space $(X, d)$ is called a $\mathrm{UC}$ space if every real-valued continuous function on $(X, d)$ is uniformly continuous. According to [13], the study of UC spaces can be traced back at least to 1947 [14]. Then Nagata studied such spaces in 1950 in [25], while in 1958 several new equivalent characterizations of such spaces were studied by Atsuji in [3]. The UC spaces have been studied by many authors [4, 5, 9]. Moreover, a wide collection of equivalent conditions for a metric space to be a UC space can be found in the survey article [24] by Kundu and Jain.

For discussing completeness of a metric space, one has to consider its corresponding Cauchy sequences. In 1971, Howes [19] generalized the notion of Cauchy sequences in terms of nets, which he called cofinally Cauchy; replacing "residual" by "cofinal" in the definition of Cauchy sequences, we obtain sequences that are called

https://doi.org/10.5186/aasfm.2020.4542

2010 Mathematics Subject Classification: Primary 54E40, 26A16, 26A99; Secondary 26A15, 54E50, 54D35.

Key words: Cofinally Cauchy regular (CC-regular) function, locally Lipschitz function, cofinally complete, UC space, uniformly locally Lipschitz function, cofinally Bourbaki-Cauchy regular (CBC-regular) function, pseudo-Cauchy regular (PC-regular) function. 
cofinally Cauchy. More precisely, a sequence $\left(x_{n}\right)$ in a metric space $(X, d)$ is said to be cofinally Cauchy if for each $\epsilon>0$, there exists an infinite subset $\mathbf{N}_{\epsilon}$ of $\mathbf{N}$ such that for each $n, j \in \mathbf{N}_{\epsilon}$, we have $d\left(x_{n}, x_{j}\right)<\epsilon$. A metric space is said to be cofinally complete if each cofinally Cauchy sequence in it has a cluster point. A metric space is called uniformly paracompact [26] if for each open cover $\mathcal{V}$ of X, there exists an open refinement $\mathcal{U}$ and $\delta>0$ such that for each $x \in X, B(x, \delta)$ intersects only finitely many members of $\mathcal{U}$. It is well-known that uniform paracompactness and cofinal completeness are equivalent properties [27] (see also [20, p. 95]). In 2008, Beer [6] gave a new direction to the study of cofinal complete metric spaces by adding their various nice and useful characterizations. Actually, the class of cofinally complete metric spaces lies between the class of complete metric spaces and that of UC spaces. Motivated by the significance of Cauchy-continuous functions [29, 28], in 2016, Aggarwal and Kundu [2] defined another class of functions called cofinally Cauchy regular (or CC-regular for short). A function $f:(X, d) \rightarrow(Y, \rho)$ between two metric spaces $(X, d)$ and $(Y, \rho)$ is called Cauchy-continuous (also referred to as Cauchy-sequentially regular; CS-regular for short) if for any Cauchy sequence $\left(x_{n}\right)$ in $(X, d),\left(f\left(x_{n}\right)\right)$ is Cauchy in $(Y, \rho)$, while $f$ is called cofinally Cauchy regular (or CC-regular) if $\left(f\left(x_{n}\right)\right)$ is cofinally Cauchy in $(Y, \rho)$ for every cofinally Cauchy sequence $\left(x_{n}\right)$ in $(X, d)$. In [1], the authors gave several equivalent characterizations of a cofinally complete metric space in terms of CC-regular functions.

Functions play a significant role in the theory of metric spaces. Two important classes of functions, namely the class of continuous functions and that of uniformly continuous functions, are well-known to all of us. A function $f:(X, d) \rightarrow(Y, \rho)$ between two metric spaces $(X, d)$ and $(Y, \rho)$ is called uniformly continuous if $\forall \epsilon>$ $0, \exists \delta>0$ such that for each $A \subseteq X$ with the diameter of $A$ less than $\delta$, the diameter of $f(A)$ is less than $\epsilon$, that is, $f(A) \subseteq B(b, \epsilon)$ for some $b \in Y$. Recently in 2017, Keremedis [22] generalized the defintion of uniformly continuous functions and apparently defined a new class of functions which he called almost bounded. In the definition of uniformly continuous functions, if we replace one open ball of radius $\epsilon$ in the metric space $Y$ by a union of finitely many open balls of radius $\epsilon$ we get the definition of almost bounded functions. More precisely, a function $f$ from a metric space $(X, d)$ to a metric space $(Y, \rho)$ is called almost bounded if for $\forall \epsilon>0, \exists \delta>0$ such that for each $A \subseteq X$ with the diameter of $A$ less than $\delta$, there exists a finite subset $B=\left\{b_{1}, b_{2}, \ldots, b_{n}\right\}$ of $Y$ such that $f(A) \subseteq \bigcup_{i=1}^{n} B\left(b_{i}, \epsilon\right)$. But an almost bounded function need not be continuous. Hence Keremedis considered those functions which are continuous as well as almost bounded and called them almost uniformly continuous. He also cast light on those metric spaces on which every continuous function is almost uniformly continuous and called such metric spaces to be almost uniformly continuous spaces or AUC spaces for short. The class of AUC spaces lies strictly in between the class of Atsuji spaces and the class of complete metric spaces.

In Section 2 of this paper, we study the relation between cofinally complete metric spaces and AUC spaces through certain functions. In fact, we show that an almost bounded function between two metric spaces is nothing but a CC-regular function and an AUC space is nothing but a cofinally complete metric space. Consequently an almost uniformly continuous function between two metric spaces is nothing but a continuous CC-regular function.

In view of this result, in Section 3, we explore some interesting properties of continuous CC-regular functions. Recently in [2], the authors have studied several 
interesting classes of bounded real-valued functions on metric spaces in order to characterize some particular families of metric spaces. In order to study boundedness of continuous CC-regular functions, we characterize those metric spaces on which every such function is bounded. Interestingly such metric spaces are same as totally bounded metric spaces. We also study boundedness of various combinations of Lipschitz-type functions with CC-regular functions. We discuss a few properties of these functions and give some examples in the context of their relation with each other. Also, using these combinations of functions, we present some new characterizations of cofinally complete metric spaces and UC spaces. Here we should mention that the metric spaces on which every real-valued uniformly continuous function is bounded are precisely those which are finitely chainable, see [23]. On the other hand, the metric spaces on which every real-valued continuous function is bounded are precisely those which are compact.

To characterize finite chainability sequentially, Garrido and Meroño defined Bourbaki-Cauchy and cofinally Bourbaki-Cauchy sequences [17]. Corresponding to these sequences, Garrido and Meroño have introduced two new types of complete metric spaces, namely Bourbaki-complete metric spaces and cofinally Bourbaki-complete metric spaces. A metric space (X, d) is said to be (cofinally) Bourbaki-complete if every (cofinally) Bourbaki-Cauchy sequence in X clusters. The concept of cofinally Bourbaki-Cauchy sequences is similar to that of cofinally Cauchy sequences, in fact, every cofinally Bourbaki-complete metric space is cofinally complete. Thus in Section 4, we study the functions which preserve cofinally Bourbaki-Cauchy sequences. We study some equivalent characterizations of such functions, their boundedness together with some Lipschitz-type functions and also find their relation with CC-regular functions.

The symbols $\mathbf{R}$ and $\mathbf{N}$ denote the sets of real numbers and natural numbers respectively. Unless mentioned otherwise $\mathbf{R}$ and its non-empty subsets carry the usual distance metric and all metric spaces are infinite. If $(X, d)$ is a metric space, $x \in X$ and $\delta>0$, then $B(x, \delta)$ denotes the open ball in $(X, d)$, centered at $x$ with radius $\delta$. Also, $(\widehat{X}, d)$ denotes the completion of a metric space $(X, d)$ and $d(A)$ denotes the diameter of a subset $A$ of $X$.

\section{CC-regular functions vis-á-vis almost bounded functions}

In this section, we study the relation between cofinally complete metric spaces and AUC spaces. In fact, we show that an almost bounded function between two metric spaces is nothing but a CC-regular function. Consequently an almost uniformly continuous function between two metric spaces is nothing but a continuous CCregular function. Let us first recall a few relevant definitions.

Definitions 2.1. A sequence $\left(x_{n}\right)$ in a metric space $(X, d)$ is called cofinally Cauchy if $\forall \epsilon>0$, there exists an infinite subset $\mathbf{N}_{\epsilon}$ of $\mathbf{N}$ such that for each $n, j \in \mathbf{N}_{\epsilon}$, we have $d\left(x_{n}, x_{j}\right)<\epsilon$. A metric space $(X, d)$ is said to be cofinally complete if every cofinally Cauchy sequence in $X$ clusters, while $(X, d)$ is called a $U C$ space if every real-valued continuous function on $X$ is uniformly continuous.

Definitions 2.2. A function $f:(X, d) \rightarrow(Y, \rho)$ between two metric spaces is said to be:

(a) Cauchy-continuous if $\left(f\left(x_{n}\right)\right)$ is Cauchy in $(Y, \rho)$ for every Cauchy sequence $\left(x_{n}\right)$ in $(X, d)$. 
(b) cofinally Cauchy regular (or CC-regular) if $\left(f\left(x_{n}\right)\right)$ is cofinally Cauchy in $(Y, \rho)$ for every cofinally Cauchy sequence $\left(x_{n}\right)$ in $(X, d)$.

(c) uniformly locally bounded if $\exists \delta>0$ such that $\forall x \in X, g\left(B_{d}(x, \delta)\right)$ is a bounded subset of $(Y, \rho)$.

Definitions 2.3. [22] A function $f$ from a metric space $(X, d)$ to a metric space $(Y, \rho)$ is called almost bounded if $\forall \epsilon>0, \exists \delta>0$ such that for every $A \subseteq X$ with $d(A)<\delta$, there exists a finite subset $B=\left\{b_{1}, b_{2}, \ldots, b_{n}\right\}$ of $Y$ such that $f(A) \subseteq$ $\bigcup_{i=1}^{n} B\left(b_{i}, \epsilon\right)$. Moreover, a continuous almost bounded function from a metric space $(X, d)$ to a metric space $(Y, \rho)$ is called almost uniformly continuous. A metric space $(X, d)$ is said to be an almost uniformly continuous space or an $A U C$ space if every continuous real-valued function on $(X, d)$ is almost uniformly continuous.

Remark 2.4. An almost bounded function between two metric spaces need not be continuous. For example, consider $X=\{0\} \cup\{1 / n: n \in \mathbf{N}\}$ with the usual distance metric and define $g(1 / n)=1 \forall n \in \mathbf{N}$ and $g(0)=0$. Also, every uniformly continuous function between two metric spaces is almost uniformly continuous, but an almost uniformly continuous function need not be even Cauchy-continuous. For example, consider $X=\left\{\frac{1}{n}: n \in \mathbf{N}\right\}$ with the usual distance metric and let $f: X \rightarrow \mathbf{R}$ be defined as:

$$
f\left(\frac{1}{n}\right)= \begin{cases}1, & n \text { is odd } \\ 2, & n \text { is even }\end{cases}
$$

In [6], Beer provided a nice equivalent characterization of cofinally complete metric spaces: A metric space $(X, d)$ is cofinally complete if and only if each continuous function from $(X, d)$ to any metric space $(Y, \rho)$ is uniformly locally bounded. In fact, in [1], the authors gave another useful equivalent characterization of cofinally complete metric spaces: A metric space $(X, d)$ is cofinally complete if and only if each continuous function from $(X, d)$ to any metric space $(Y, \rho)$ is $C C$-regular.

Now we state relations between the above defined functions which are used to define AUC spaces and characterize cofinally complete spaces. Consequently, we get relation between AUC spaces and cofinally complete spaces.

Theorem 2.5. If $f$ is a $C C$-regular function from a metric space $(X, d)$ to another metric space $(Y, \rho)$, then $f$ is uniformly locally bounded.

Proof. Let $f:(X, d) \rightarrow(Y, \rho)$ be a CC-regular function. Suppose $f$ is not uniformly locally bounded. Therefore $\forall n \in \mathbf{N}, \exists x_{n} \in X$ such that $f\left(B\left(x_{n}, \frac{1}{n}\right)\right)$ is not a bounded subset of $Y$. Let $\epsilon$ be any positive real number. Now, $\forall n \in \mathbf{N}$, $f\left(B\left(x_{n}, \frac{1}{n}\right)\right)$ is not bounded in $Y$. Therefore, $\forall n \in \mathbf{N}$, there exits a sequence $\left(f\left(x_{n}^{m}\right)\right)_{m \in \mathbf{N}}$ in $f\left(B\left(x_{n}, \frac{1}{n}\right)\right)$ such that $\rho\left(f\left(x_{n}^{m}\right), f\left(x_{n}^{t}\right)\right) \geq \epsilon$ for all $m, t \in \mathbf{N}$. Let $A_{n}=\left\{f\left(x_{n}^{m}\right): m \in \mathbf{N}\right\}$. Now we will construct a sequence in $Y$ by induction. Let $F_{1}=\left\{f\left(x_{1}^{1}\right)\right\}$. If $d\left(x_{1}^{1}, y\right)<\frac{\epsilon}{2}$ for every $y \in A_{2}$, then $d\left(y, y^{\prime}\right)<\epsilon$ for all $y, y^{\prime} \in A_{2}$. We get a contradiction. Let $f\left(x_{2}^{2}\right)$ (rename if necessary) $\in A_{2}$ such that $\rho\left(f\left(x_{1}^{1}\right), f\left(x_{2}^{2}\right)\right) \geq \frac{\epsilon}{2}$. Similarly, we can choose $f\left(x_{1}^{2}\right) \in A_{1} \backslash\left\{f\left(x_{1}^{1}\right), f\left(x_{2}^{2}\right)\right\}$ such that $F_{2}=\left\{f\left(x_{1}^{1}\right), f\left(x_{2}^{2}\right), f\left(x_{1}^{2}\right)\right\}$ is $\frac{\epsilon}{2}$ discrete. Now suppose a finite subset $F_{n}$ of $\bigcup_{i=1}^{n} A_{i}$ is chosen satisfying: 1) $F_{n}$ is $\frac{\epsilon}{2}$ discrete, 2) $\left|F_{n} \cap A_{i}\right|=n-i+1$ for all $1 \leq i \leq n$. To construct $F_{n+1}$, choose $f\left(x_{n+1}^{n+1}\right) \in A_{n+1}$ such that $F_{n} \cup\left\{f\left(x_{n+1}^{n+1}\right)\right\}$ is $\frac{\epsilon}{2}$ discrete. Suppose it is not possible, then $\rho\left(y, F_{n}\right)<\frac{\epsilon}{2}$ for all $y \in A_{n+1}$. Since $A_{n+1}$ is infinite and $F_{n}$ is finite, there exists $y, y^{\prime} \in A_{n+1} \backslash F_{n}, y \neq y^{\prime}$ such that $\rho(y, z)<\frac{\epsilon}{2}$ and $\rho\left(y^{\prime}, z\right)<\frac{\epsilon}{2}$ for some $z \in F_{n}$. This implies $\rho\left(y, y^{\prime}\right)<\epsilon$, but it gives a contradiction. Repeating this 
process, we construct $F_{n+1}=F_{n} \bigcup\left\{f\left(x_{1}^{n+1}\right), f\left(x_{2}^{n+1}\right), \ldots f\left(x_{n_{+}}^{n+1}\right)\right\}$ such that $F_{n+1}$ is $\frac{\epsilon}{2}$ discrete. Now if we take a sequence in the order we chose the elements of the type $f\left(x_{j}^{i}\right), i, j \in \mathbf{N}$, the sequence is $\frac{\epsilon}{2}$ discrete but its pre-image is cofinally Cauchy as it consists of infinite elements from each ball $B\left(x_{n}, \frac{1}{n}\right)$. Since $f$ is CC-regular, we get a contradiction.

Remark 2.6. A uniformly locally bounded function between two metric spaces need not be CC-regular. For example, consider $X=\left\{\frac{1}{n}: n \in \mathbf{N}\right\}$ with the usual distance metric $d$ and $Y=\{n: n \in \mathbf{N}\}$ with the $\{0,1\}$ discrete metric $\rho$. Define $f$ from $(X, d)$ to $(Y, \rho)$ such that $f\left(\frac{1}{n}\right)=n$ for all $n \in \mathbf{N}$.

Note that the above example shows that even a continuous uniformly locally bounded function need not be CC-regular. But for a special kind of range, we have the following result.

Proposition 2.7. Let $f:(X, d) \rightarrow(Y, \rho)$ be a function between two metric spaces. Suppose every bounded subset of $Y$ is totally bounded. Then $f$ is $C C$-regular if and only if it is uniformly locally bounded.

Theorem 2.8. Let $f$ be a function between two metric spaces $(X, d)$ and $(Y, \rho)$. Then $f$ is CC-regular if and only if $f$ is almost bounded.

Proof. In a manner similar to the proof of Theorem 2.5, we can prove that every CC-regular function between two metric spaces is almost bounded. Conversely, let $f:(X, d) \rightarrow(Y, \rho)$ be an almost bounded function and let $\left(x_{n}\right)$ be a cofinally Cauchy sequence in $X$ having no constant subsequence. Let $\epsilon>0$, then there exists a $\delta>$ 0 such that for all $A \subseteq X$ with $d(A)<\delta$, there exists $\left\{y_{1}, y_{2}, \ldots, y_{n}\right\} \subseteq Y$ such that $f(A) \subset \bigcup_{i=1}^{n} B\left(y_{i}, \epsilon\right)$. Since $\left(x_{n}\right)$ is cofinally Cauchy, there exists an infinite subset $\mathbf{N}_{\delta}$ of $\mathbf{N}$ such that $d\left(x_{n}, x_{m}\right)<\delta$ for all $n, m \in \mathbf{N}_{\delta}$. Let $A=\left\{x_{n}: n \in \mathbf{N}_{\delta}\right\}$. Thus $f(A) \subset \bigcup_{i=1}^{n} B\left(y_{i}, \epsilon\right)$ for some $y_{i} \in Y, 1 \leq i \leq n$. Thus, there exists $i \in \mathbf{N}$ such that $B\left(y_{i}, \epsilon\right)$ contains elements of the type $f\left(x_{k}\right)$ for infinitely many $k \in \mathbf{N}$. Hence the sequence $\left(f\left(x_{n}\right)\right)$ is cofinally Cauchy.

Remark 2.9. Since CC-regular functions are more familiar in the literature, from now onwards we will call almost bounded functions to be CC-regular functions.

Corollary 2.10. A metric space $(X, d)$ is an AUC space if and only if it is cofinally complete.

Proof. Since the class of CC-regular functions and that of almost bounded functions are same, by the definition of AUC spaces and by the fact that a metric space $(X, d)$ is cofinally complete if and only if each real-valued continuous function defined on it is CC-regular [1], we conclude that a metric space $(X, d)$ is an AUC space if and only if it is cofinally complete.

Remark 2.11. It is easy to see that a CC-regular function between two metric spaces need not be bounded; consider identity function on $\mathbf{R}$ with the usual distance metric. Also note that a bounded function need not be CC-regular. For example, consider $X=\left\{\frac{1}{n}: n \in \mathbf{N}\right\}$ with the usual distance metric $d$ and $Y=\mathbf{N}$ with the $\{0,1\}$ discrete metric $d^{\prime}$. Let $f:(X, d) \rightarrow\left(Y, d^{\prime}\right)$ be defined as:

$$
f\left(\frac{1}{n}\right)=n \quad \forall n \in \mathbf{N} .
$$


In the literature one can find another important generalization of Cauchy sequences (other than cofinally Cauchy sequences), known as pseudo-Cauchy sequences. These sequences play an important role in characterizing UC spaces (discovered by Toader [30]): a metric space is UC if and only if each pseudo-Cauchy sequence of distinct points in the space clusters. In [2], the authors studied those functions which preserve pseudo-Cauchy sequences (called PC-regular functions) and characterized UC space as follows: a metric space is UC if and only if each real-valued continuous function defined on it is PC-regular. Our next result gives some equivalent characterizations of totally bounded metric spaces using CC-regular and PC-regular functions. Before that we need to recall the following definitions.

Definitions 2.12. A sequence $\left(x_{n}\right)$ in a metric space $(X, d)$ is said to be pseudoCauchy if $\forall \epsilon>0$ and $n \in \mathbf{N}, \exists k, j \in \mathbf{N}$ such that $k, j>n, k \neq j$ and $d\left(x_{k}, x_{j}\right)<\epsilon$. A function $f:(X, d) \rightarrow(Y, \rho)$ between two metric spaces is said to be pseudo-Cauchy regular (or PC-regular for short) if $\left(f\left(x_{n}\right)\right)$ is pseudo-Cauchy in $(Y, \rho)$ for every pseudo-Cauchy sequence $\left(x_{n}\right)$ in $(X, d)$.

Theorem 2.13. Let $(Y, \rho)$ be a metric space. The following statements are equivalent:

(a) $(Y, \rho)$ is totally bounded.

(b) If $(X, d)$ is a metric space such that $(\widehat{X}, d)$ has an accumulation point, then every function $f:(X, d) \rightarrow(Y, \rho)$ is $P C$-regular.

(c) If $(X, d)$ is a metric space such that $(\widehat{X}, d)$ has an accumulation point, then every function $f:(X, d) \rightarrow(Y, \rho)$ is $C C$-regular.

(d) There exists a metric space $(X, d)$ such that $(\widehat{X}, d)$ has an accumulation point and every function $f:(X, d) \rightarrow(Y, \rho)$ is $C C$-regular.

Proof. $(\mathrm{a}) \Rightarrow(\mathrm{b})$ : Let $(X, d)$ be a metric space such that $(\widehat{X}, d)$ has an accumulation point. Since $Y$ is totally bounded, every sequence in $(Y, \rho)$ is pseudo-Cauchy. Hence every function $f:(X, d) \rightarrow(Y, \rho)$ is PC-regular.

(b) $\Rightarrow(c)$ : This follows from the fact that every PC-regular function between any two metric spaces is CC-regular [2].

$(c) \Rightarrow(d)$ : This is immediate.

$(\mathrm{d}) \Rightarrow(\mathrm{a})$ : Let $(X, d)$ be a metric space such that $(\widehat{X}, d)$ has an accumulation point and every function $f:(X, d) \rightarrow(Y, \rho)$ is CC-regular. Suppose $(Y, \rho)$ is not totally bounded. Thus there exists a sequence $\left(y_{n}\right)$ in $Y$ and $\epsilon>0$ such that $\rho\left(y_{i}, y_{j}\right) \geq \epsilon$ for all $i, j \in \mathbf{N}$. Since the set of limit points of $(\widehat{X}, d)$ is non-empty, there exists a Cauchy sequence of distinct terms $\left(\hat{x}_{n}\right)$ in $\hat{X}$. Thus there exists a sequence $\left(x_{n}\right)$ in $X$ consisting of distinct terms such that $d\left(x_{n}, \hat{x}_{n}\right)<1 / n$ for all $n \in \mathbf{N}$. Thus, $\left(x_{n}\right)$ is Cauchy in $X$. Define a function $f:(X, d) \rightarrow(Y, \rho)$ as follows:

$$
f(x)= \begin{cases}y_{n} & \text { if } x=x_{n} \text { for some } n \\ y_{1} & \text { otherwise }\end{cases}
$$

According to the hypothesis, $f$ should be CC-regular, but $\left(x_{n}\right)$ is cofinally Cauchy in $X$ such that $\left(f\left(x_{n}\right)\right)$ is not cofinally Cauchy. We get a contradiction. Thus $(Y, \rho)$ is totally bounded. 


\section{Continuous CC-regular functions}

In this section, we study continuous CC-regular functions and in the process, we obtain several new interesting results. Several new characterizations of totally bounded metric spaces, cofinally complete metric spaces and UC spaces are also obtained.

It is well-known that continuous functions are characterized by convergent sequences, whereas uniformly continuous functions are characterized by asymptotic sequences. In [1], the authors generalized the notion of asymptotic sequences. Before proceeding further, let us recall a few definitions.

Definitions 3.1. A pair of sequences $\left(x_{n}\right)$ and $\left(y_{n}\right)$ in a metric space $(X, d)$ is said to be:

(a) asymptotic, written $\left(x_{n}\right) \asymp\left(y_{n}\right)$, if $\forall \epsilon>0, \exists n_{o} \in \mathbf{N}$ such that $d\left(x_{n}, y_{n}\right)<\epsilon$ $\forall n>n_{o}$.

(b) uniformly asymptotic, written $\left(x_{n}\right) \asymp^{u}\left(y_{n}\right)$, if $\forall \epsilon>0, \exists n_{o} \in \mathbf{N}$ such that $d\left(x_{m}, y_{n}\right)<\epsilon \forall m, n>n_{o}$.

(c) cofinally asymptotic, written $\left(x_{n}\right) \asymp_{c}\left(y_{n}\right)$, if $\forall \epsilon>0, \exists$ an infinite subset $N_{\epsilon}$ of $\mathbf{N}$ such that $d\left(x_{n}, y_{n}\right)<\epsilon \forall n \in N_{\epsilon}$.

(d) cofinally uniformly asymptotic, written $\left(x_{n}\right) \asymp_{c}^{u}\left(y_{n}\right)$, if $\forall \epsilon>0, \exists$ an infinite subset $N_{\epsilon}$ of $\mathbf{N}$ such that $d\left(x_{n}, y_{m}\right)<\epsilon \forall n, m \in N_{\epsilon}$.

In [28], Snipes gave a nice characterization of Cauchy-continuous functions in terms of pairs of uniformly asymptotic sequences: a function $f:(X, d) \rightarrow(Y, \rho)$ between two metric spaces is Cauchy-continuous if and only if $\left(x_{n}\right) \asymp^{u}\left(z_{n}\right)$ in $(X, d)$ implies $\left(f\left(x_{n}\right)\right) \asymp^{u}\left(f\left(z_{n}\right)\right)$ in $(Y, \rho)$. Note that a sequence $\left(x_{n}\right)$ in $(X, d)$ is cofinally Cauchy if and only if $\left(x_{n}\right) \asymp_{c}^{u}\left(x_{n}\right)$. Thus if $f:(X, d) \rightarrow(Y, \rho)$ is a continuous function that preserves cofinally uniformly asymptotic sequences (that is, $\left(x_{n}\right) \asymp_{c}^{u}\left(z_{n}\right)$ implies $\left.\left(f\left(x_{n}\right)\right) \asymp_{c}^{u} f\left(\left(z_{n}\right)\right)\right)$, then $\mathrm{f}$ is CC-regular, but the converse is not true.

Example 3.2. Consider $A=\left\{\frac{1}{n}: n \in \mathbf{N}\right\}$ with the usual distance metric and let $f: A \rightarrow \mathbf{R}$ be defined as:

$$
f(1 / n)= \begin{cases}1, & n \text { is odd } \\ 2, & n \text { is even }\end{cases}
$$

Clearly, $f$ is continuous and CC-regular, but $f$ does not preserve cofinally uniformly asymptotic sequences (for example, take $x_{n}=\frac{1}{2 n-1}$ and $z_{n}=\frac{1}{2 n}$ ).

Note that the above function also shows that unlike Cauchy-continuous functions, a continuous CC-regular function on a totally bounded metric space need not be uniformly continuous.

In [1], the authors have given the following sequential characterization of CCregular functions.

Proposition 3.3. [1, Proposition 2.11] Let $f:(X, d) \rightarrow(Y, \rho)$ be a function between two metric spaces. The following statements are equivalent:

(a) $f$ is $C C$-regular.

(b) If $\left(x_{n}\right)$ is a cofinally Cauchy sequence in $(X, d)$ and $\left(f\left(x_{n}\right)\right) \asymp\left(f\left(z_{n}\right)\right)$, where $\left(z_{n}\right)$ is any sequence in $X$, then $\left(f\left(x_{n}\right)\right) \asymp_{c}^{u}\left(f\left(z_{n}\right)\right)$.

(c) If $\left(x_{n}\right) \asymp_{c}^{u}\left(z_{n}\right)$ and $\left(f\left(x_{n}\right)\right) \asymp\left(f\left(z_{n}\right)\right)$, then $\left(f\left(x_{n}\right)\right) \asymp_{c}^{u}\left(f\left(z_{n}\right)\right)$.

Remark 3.4. Note that if we replace $\left(f\left(x_{n}\right)\right) \asymp\left(f\left(z_{n}\right)\right)$ by $\left(f\left(x_{n}\right)\right) \asymp_{c}\left(f\left(z_{n}\right)\right)$ in $(b)$, still the condition will imply the function to be CC-regular but the converse 
may not be true. For example, consider the identity function on $X=\left\{n, \frac{1}{n}: n \in \mathbf{N}\right\}$ endowed with the usual distance metric. Let $\left(x_{n}\right)$ be the sequence $1, \frac{1}{2}, 2, \frac{1}{3}, 3, \frac{1}{4}, 4, \ldots$ and $\left(z_{n}\right)$ be the sequence $1,10,2,10,3,10,4, \ldots$, here the function is continuous and CC-regular, and $\left(x_{n}\right)$ is cofinally Cauchy such that $\left(f\left(x_{n}\right)\right) \asymp_{c}\left(f\left(z_{n}\right)\right)$ but $\left(f\left(x_{n}\right)\right) \varkappa_{c}^{u}$ $\left(f\left(z_{n}\right)\right)$.

In order to characterize continuous CC-regular functions using sequences, we observe the following sequential characterization of continuous functions.

Proposition 3.5. Let $f:(X, d) \rightarrow(Y, \rho)$ be a function between two metric spaces. The following statements are equivalent:

(a) $f$ is continuous.

(b) Whenever $\left(x_{n}\right) \asymp_{c}(x)$ in $(X, d)$, then $\left(f\left(x_{n}\right)\right) \asymp_{c}(f(x))$ in $(Y, \rho)$.

(c) Whenever $\left(x_{n}\right) \asymp(x)$ in $(X, d)$, then $\left(f\left(x_{n}\right)\right) \asymp_{c}(f(x))$ in $(Y, \rho)$.

Proof. $(\mathrm{a}) \Rightarrow(\mathrm{b})$ : Since $\left(x_{n}\right) \asymp_{c}(x)$, there exists a subsequence of $\left(x_{n}\right)$ which converges to $x$. Using the continuity of $f,\left(f\left(x_{n}\right)\right) \asymp_{c}(f(x))$.

(b) $\Rightarrow(\mathrm{c})$ : This is immediate.

(c) $\Rightarrow$ (a): Suppose $f$ is not continuous at $x$. Thus, $\exists \epsilon>0$ such that $\forall n \in$ $\mathbf{N}, \exists x_{n}$ such that $d\left(x_{n}, x\right)<\frac{1}{n}$ but $\rho\left(f\left(x_{n}\right), f(x)\right) \geq \epsilon$. We get a contradiction.

Now the following sequential characterization of continuous CC-regular functions is immediate.

Proposition 3.6. Let $f:(X, d) \rightarrow(Y, \rho)$ be a function between two metric spaces. The following are equivalent:

(a) $f$ is continuous and CC-regular.

(b) If $\left(x_{n}\right)$ is a cofinally Cauchy sequence and $\left(f\left(x_{n}\right)\right) \asymp\left(f\left(z_{n}\right)\right)$, then $\left(f\left(x_{n}\right)\right) \asymp_{c}^{u}$ $\left(f\left(z_{n}\right)\right)$. Also, if $\left(y_{n}\right) \asymp_{c}(y)$, then $\left(f\left(y_{n}\right)\right) \asymp_{c}(f(y))$.

(c) If $\left(x_{n}\right) \asymp_{c}^{u}\left(z_{n}\right)$ and $\left(f\left(x_{n}\right)\right) \asymp\left(f\left(z_{n}\right)\right)$, then $\left(f\left(x_{n}\right)\right) \asymp_{c}^{u}\left(f\left(z_{n}\right)\right)$. Also, if $\left(y_{n}\right) \asymp(y)$, then $\left(f\left(y_{n}\right)\right) \asymp_{c}(f(y))$.

We know that every continuous function on a metric space is CC-regular if and only if the metric space is cofinally complete. Our next result characterizes those metric spaces $(X, d)$ such that every CC-regular function from $(X, d)$ to any metric space $(Y, \rho)$ is continuous. Such metric spaces are also equivalent to those metric spaces on which every PC-regular function is continuous.

Theorem 3.7. Let $(X, d)$ be a metric space. Then the following are equivalent:

(a) The metric space $(X, d)$ is discrete.

(b) Whenever $(Y, \rho)$ is a metric space and $f:(X, d) \rightarrow(Y, \rho)$ is $C C$-regular, then $f$ is continuous.

(c) Whenever $(Y, \rho)$ is a metric space and $f:(X, d) \rightarrow(Y, \rho)$ is PC-regular, then $f$ is continuous.

Proof. The implications (a) $\Rightarrow(\mathrm{b}) \Rightarrow$ (c) are immediate.

$(c) \Rightarrow(a)$ : Suppose $(X, d)$ is not discrete. Then there exists a non-isolated point $x$ in $X$. Hence there exists a sequence $\left(x_{n}\right)$ in $X$ such that all the elements of the sequence are distinct, $x_{n} \neq x \forall n \in \mathbf{N}$ and $\left(x_{n}\right)$ converges to $x \in X$. Define $f:(X, d) \rightarrow \mathbf{R}$ such that

$$
f(z)= \begin{cases}1, & \text { if } z=x_{n} \text { for some } n \\ 0, & \text { otherwise }\end{cases}
$$


Clearly, $f$ is PC-regular but not continuous.

Analogously we can characterize those metric spaces on which every CC-regular and PC-regular functions are Cauchy continuous and uniformly continuous. Every Cauchy continuous function on a metric space $(X, d)$ is CC-regular if and only if $(\widehat{X}, d)$ is cofinally complete and every Cauchy continuous function on $(X, d)$ is PCregular if and only if $(\widehat{X}, d)$ is UC space [1]. Also, in the same article it has been proved that for a metric space $(X, d)$, every CC-regular function on it with values in any arbitrary metric space $(Z, \rho)$ is uniformly continuous if and only if $(X, d)$ is uniformly discrete.

Theorem 3.8. Let $(X, d)$ be a metric space. Then the following are equivalent:

(a) The metric space $(X, d)$ is complete and discrete.

(b) Whenever $(Y, \rho)$ is a metric space and $f:(X, d) \rightarrow(Y, \rho)$ is CC-regular, then $f$ is Cauchy continuous.

(c) Whenever $(Y, \rho)$ is a metric space and $f:(X, d) \rightarrow(Y, \rho)$ is PC-regular, then $f$ is Cauchy continuous.

Proof. $(\mathrm{a}) \Rightarrow(\mathrm{b})$ : Let $f:(X, d) \rightarrow(Y, \rho)$ be CC-regular function. Since $(X, d)$ is discrete, $f$ is continuous, thus Cauchy continuous because $(X, d)$ is complete.

(b) $\Rightarrow$ (c): This is immediate.

$\left(\right.$ c) $\Rightarrow\left(\right.$ a): Suppose there exists a Cauchy sequence $\left(x_{n}\right)$ in $(X, d)$ such that all its elements are distinct and it does not converge. Define $f:(X, d) \rightarrow \mathbf{R}$ such that

$$
f(x)= \begin{cases}1, & \text { if } x=x_{n} \text { for some odd } n \\ 2, & \text { if } x=x_{n} \text { for some even } n \\ 0, & \text { otherwise }\end{cases}
$$

Clearly $f$ is PC-regular but not Cauchy continuous. Thus $(X, d)$ is complete and by Theorem 3.7, $(X, d)$ is discrete.

Theorem 3.9. Let $(X, d)$ be a metric space. Then the following are equivalent:

(a) The metric space $(X, d)$ is uniformly discrete.

(b) Whenever $(Y, \rho)$ is a metric space and $f:(X, d) \rightarrow(Y, \rho)$ is CC-regular, then $f$ is uniformly continuous.

(c) Whenever $(Y, \rho)$ is a metric space and $f:(X, d) \rightarrow(Y, \rho)$ is PC-regular, then $f$ is uniforly continuous.

Proof. The implications (a) $\Rightarrow(\mathrm{b}) \Rightarrow$ (c) are immediate.

$(\mathrm{c}) \Rightarrow(\mathrm{a})$ : In a manner similar to the proof of Theorem 2.8 in [1], we can prove the result.

It is well-known that the set of all real-valued continuous functions defined on a metric space $(X, d)$ forms a ring with respect to the usual addition and multiplication of real-valued functions, but this is not true in case of uniformly continuous functions [11]. We have the following nice observation regarding continuous CC-regular functions.

Theorem 3.10. Let $(X, d)$ be a metric space and let $A$ be the set of all realvalued continuous $C C$-regular functions on $X$. Then $A$ is a ring with respect to the usual addition and multiplication of real-valued functions.

Proof. A function $f:(X, d) \rightarrow \mathbf{R}$ is CC-regular if and only if it is uniformly locally bounded (see Proposition 2.7). Let $f, g:(X, d) \rightarrow \mathbf{R}$ be two continuous 
CC-regular functions. So there exists a $\delta>0$ such that $\forall x \in X, f(B(x, \delta))$ and $g(B(x, \delta))$ are bounded subsets of $\mathbf{R}$. Thus $(f+g)(B(x, \delta))$ and $(f * g)(B(x, \delta))$ are also bounded subsets of $\mathbf{R}$. Thus $f+g$ and $f * g$ are also continuous CC-regular functions. Hence the set of all real-valued continuous CC-regular functions on $X$ forms a ring with respect to the usual addition and multiplication of real-valued functions.

In analysis, there is a well-known form of continuity which is even stronger than uniform continuity, namely Lipschitz continuity. In [8, 9, 10, 16], Beer, Garrido and Jaramillo considered various functions of Lipschitz-type, the definitions of which are recalled below.

Definitions 3.11. A function $f:(X, d) \rightarrow(Y, \rho)$ between two metric spaces is said to be:

(a) Lipschitz if there exists $k>0$ such that $\rho\left(f(x), f\left(x^{\prime}\right)\right) \leq k d\left(x, x^{\prime}\right)$.

(b) Lipschitz in the small if there exist $\delta>0$ and $k>0$ such that $\rho\left(f(x), f\left(x^{\prime}\right)\right) \leq$ $k d\left(x, x^{\prime}\right)$, whenever $d\left(x, x^{\prime}\right)<\delta$.

(c) uniformly locally Lipschitz if there exists $\delta>0$ such that for every $x \in X$, there exists $k_{x}>0$ such that $\rho(f(u), f(w)) \leq k_{x} d(u, w)$, whenever $u, w \in$ $B(x, \delta)$.

(d) Cauchy-Lipschitz if $f$ is Lipschitz when restricted to the range of each Cauchy sequence $\left(x_{n}\right)$ in $X$.

(e) locally Lipschitz if for each $x \in X$, there exists $\delta_{x}>0$ such that $f$ restricted to $B\left(x, \delta_{x}\right)$ is Lipschitz.

It is immediate that every Lipschitz in the small function between two metric spaces is uniformly locally Lipschitz. Moreover, it is shown in [10] that the collection of Cauchy-Lipschitz functions is contained in the class of locally Lipschitz functions where the collection itself contains the uniformly locally Lipschitz functions. But the reverse implications are not in general true. In [11], it has been proved that a metric space $(X, d)$ is $\mathrm{UC}$ if and only if the reciprocal of each never zero real-valued uniformly continuous function is uniformly continuous. We now find some analogous conditions in terms of CC-regular functions, locally Lipschitz functions and PC-regular functions for cofinally complete spaces and UC spaces (see also Theorem 4.8).

Theorem 3.12. Let $(X, d)$ be a metric space. Then the following statements are equivalent:

(a) $(X, d)$ is a cofinally complete metric space.

(b) Whenever $f:(X, d) \rightarrow \mathbf{R}$ is a locally Lipschitz CC-regular function such that $f$ is never zero, then $\frac{1}{f}$ is also locally Lipschitz and CC-regular.

(c) Whenever $f:(X, d) \rightarrow \mathbf{R}$ is a continuous $C C$-regular function such that $f$ is never zero, then $\frac{1}{f}$ is also continuous and CC-regular.

Proof. The implications (a) $\Rightarrow(\mathrm{b})$ and $(\mathrm{a}) \Rightarrow(\mathrm{c})$ are immediate as every realvalued continuous function on a cofinally complete metric space is CC-regular.

(b) $\Rightarrow(\mathrm{a})$ : If $(X, d)$ is not cofinally complete, then there exists a cofinally Cauchy sequence $\left(x_{n}\right)$ of distinct points in $(X, d)$ with no cluster point. Thus the set $A=$ $\left\{x_{n}: n \in \mathbf{N}\right\}$ is closed. Consequently, $\forall n \in \mathbf{N}, \exists \epsilon_{n}>0$ such that $d\left(x_{m}, x_{n}\right)>$ $\epsilon_{n} \forall m \neq n$. Let $\delta_{n}=\min \left\{1 / n, \epsilon_{n} / 3\right\}$. Define a function $f:(X, d) \rightarrow[0,2]$ as follows:

$$
f(x)= \begin{cases}\frac{1}{n}-\frac{1}{n \delta_{n}} d\left(x, x_{n}\right), & x \in B\left(x_{n}, \delta_{n}\right) \text { for some } n \in \mathbf{N}, \\ 0, & \text { otherwise. }\end{cases}
$$


Clearly, $f$ restricted to each ball $B\left(x_{n}, \delta_{n}\right)$ is Lipschitz. Let $x \in X$. Since $x$ is not a cluster point of the sequence $\left(x_{n}\right)$ and $\epsilon_{n} \leq 1 / n \forall n \in \mathbf{N}, \exists \delta_{x}>0$ such that $B\left(x, \delta_{x}\right)$ intersects at most one of the balls $B\left(x_{n}, \delta_{n}\right)$. Thus $f$ is locally Lipschitz. Since the range of $f$ is totally bounded, $f$ is CC-regular as well. Now define another function $g:(X, d) \rightarrow \mathbf{R}$ as follows:

$$
g(x)=f(x)+d(x, A)
$$

Since the sum of two real-valued locally Lipschitz functions on a metric space is also locally Lipschitz, $g$ is locally Lipschitz. Also, by Theorem 3.10, $g$ is CC-regular. By the hypothesis, $\frac{1}{g}$ should also be CC-regular since $g$ is never zero. But $\left(x_{n}\right)$ is a cofinally Cauchy sequence such that $\frac{1}{g}\left(x_{n}\right)=n \forall n \in \mathbf{N}$. We get a contradiction. Hence $(X, d)$ is cofinally complete.

$(c) \Rightarrow(a)$ : If $(X, d)$ is not cofinally complete, then there exists a cofinally Cauchy sequence $\left(x_{n}\right)$ of distinct points in $(X, d)$ with no cluster point. Thus, $A=\left\{x_{n}: n \in \mathbf{N}\right\}$ is closed and discrete. Define

$$
\begin{aligned}
& f: A \longrightarrow(0,2), \\
& x_{n} \longmapsto \frac{1}{n} .
\end{aligned}
$$

Clearly, $f$ is a continuous CC-regular function. By Theorem 5.1 in [15, p. 149], $f$ can be extended to a function $F: X \rightarrow(0,2)$, such that $F$ is continuous. Since $(0,2)$ is totally bounded in $\mathbf{R}, F$ is CC-regular. Cleary $F$ is never zero but $\frac{1}{F}$ is not CC-regular, as $\left(x_{n}\right)$ is cofinally Cauchy but $(n)$ is not cofinally Cauchy in $\mathbf{R}$. We get a contradiction.

In a manner similar to the proof of the above result, we can prove the following result.

Theorem 3.13. Let $(X, d)$ be a metric space. Then the following statements are equivalent:

(a) $(X, d)$ is a $U C$ space.

(b) Whenever $f:(X, d) \rightarrow \mathbf{R}$ is a continuous $P C$-regular function such that $f$ is never zero, then $\frac{1}{f}$ is also continuous and $P C$-regular.

Remark 3.14. Note that if the completion $(\widehat{X}, d)$ of a metric space $(X, d)$ is cofinally complete, then the following statement need not hold: whenever $f:(X, d) \rightarrow \mathbf{R}$ is a Cauchy-continuous CC-regular function such that $f$ is never zero, then $\frac{1}{f}$ is also Cauchy-continuous and CC-regular. For example, consider the identity function $f$ on $(X, d)$, where $X=\{1 / n: n \in \mathbf{N}\}$ and $d$ is the usual distance metric on $X$. The metric space $(X, d)$ has a cofinal completion. Also, $f$ is Cauchy-continuous, CC-regular and is never zero, but $\frac{1}{f}$ is not CC-regular.

We know that CC-regular and PC-regular functions between two metric spaces need not be continuous. The following example shows that a uniformly locally Lipschitz function between two metric spaces need not be CC-regular.

Example 3.15. Consider the real Hilbert space $l_{2}$. Let $X=\bigcup_{n \in \mathbf{N}} A_{n}$, where $A_{n}=\left\{e_{n}+\frac{1}{n} e_{k}: k \in \mathbf{N}\right\}$, and $\left\{e_{n}: n \in \mathbf{N}\right\}$ is the standard orthonormal basis of $l_{2}$. Let $d$ be the metric on $X$ induced by the $l_{2}$ norm. Consider $Y=\{n: n \in \mathbf{N}\}$ with the $\{0,1\}$ discrete metric $\rho$. Let $\left\{\mathbf{M}_{j}: j \in \mathbf{N}\right\}$ be a pairwise disjoint family of infinite subsets of $\mathbf{N}$. Let $\mathbf{M}_{n}=\left\{x_{1}^{n}, x_{2}^{n}, x_{3}^{n}, \ldots\right\}$ for all $n \in \mathbf{N}$. Define $f$ from $(X, d)$ 
to $(Y, \rho)$ such that $\forall k, n \in \mathbf{N}$

$$
f\left(e_{n}+\frac{1}{n} e_{k}\right)=x_{k}^{n}
$$

Now for each $z \in X$, there exists exactly one natural number $n$ such that $B(z, 1) \cap$ $A_{n} \neq \emptyset$. Choose $k_{z}>\frac{n}{\sqrt{2}}$, thus $\rho(f(u), f(w)) \leq k_{z} d(u, w)$, whenever $u, w \in B(z, 1)$. Hence the function is uniformly locally Lipschitz. The function is not CC-regular because if we enumerate the elements of $X$, we will get a cofinally Cauchy sequence but its image is certainly not cofinally Cauchy.

Remark 3.16. Since a PC-regular function between two metric spaces is CCregular, every uniformly locally Lipschitz function between two metric spaces need not be PC-regular.

We know that a metric space $(X, d)$ is compact if and only if every real-valued continuous function defined on it is bounded. Our next result characterizes in particular those metric spaces on which every real-valued continuous CC-regular function is bounded. We also study boundedness of various combinations of Lipschitz-type functions with CC-regular functions. Note that a function $f:(X, d) \rightarrow(Y, \rho)$ between two metric spaces is called bounded if $f(X)$ is bounded in $(Y, \rho)$.

Theorem 3.17. Let $(X, d)$ be a metric space. Then the following are equivalent:

(a) The metric space $(X, d)$ is totally bounded.

(b) Whenever $(Y, \rho)$ is a metric space and $f:(X, d) \rightarrow(Y, \rho)$ is continuous and $C C$-regular, then $f$ is bounded.

(c) Whenever $(Y, \rho)$ is a metric space and $f:(X, d) \rightarrow(Y, \rho)$ is locally Lipschitz and $C C$-regular, then $f$ is bounded.

(d) Whenever $(Y, \rho)$ is a metric space and $f:(X, d) \rightarrow(Y, \rho)$ is Cauchy-Lipschitz and $C C$-regular, then $f$ is bounded.

(e) Whenever $(Y, \rho)$ is a metric space and $f:(X, d) \rightarrow(Y, \rho)$ is uniformly locally Lipschitz and CC-regular, then $f$ is bounded.

(f) Whenever $f$ is a real-valued uniformly locally Lipschitz and CC-regular function on $(X, d)$, then $f$ is bounded.

Proof. The implications $(\mathrm{b}) \Rightarrow(\mathrm{c}) \Rightarrow(\mathrm{d}) \Rightarrow(\mathrm{e}) \Rightarrow(\mathrm{f})$ are all immediate.

$(\mathrm{a}) \Rightarrow(\mathrm{b})$ : Let $f$ be a continuous and CC-regular function from $(X, d)$ to $(Y, \rho)$. It is easy to see that a metric space is totally bounded if and only if every sequence in it is cofinally Cauchy. Thus $f(X)$ is a totally bounded subset of $(Y, \rho)$.

(f) $\Rightarrow(\mathrm{a})$ : Suppose $(X, d)$ is not totally bounded. Therefore, $\exists \delta>0$ and a sequence $\left(x_{n}\right)$ in $X$ such that $d\left(x_{n}, x_{m}\right)>\delta \forall n, m \in \mathbf{N}(n \neq m)$. Define a function $f:(X, d) \rightarrow \mathbf{R}$ as follows:

$$
f(x)= \begin{cases}n-\frac{4 n}{\delta} d\left(x, x_{n}\right), & x \in B\left(x_{n}, \frac{\delta}{4}\right) \text { for some } n \in \mathbf{N}, \\ 0, & \text { otherwise }\end{cases}
$$

The function $f$ is uniformly locally Lipschitz because $\forall x \in X, B\left(x, \frac{\delta}{4}\right)$ intersects at most one of the balls $B\left(x_{m}, \frac{\delta}{4}\right)$ and $f$ restricted to each ball $B\left(x_{m}, \frac{\delta}{4}\right)$ is Lipschitz.

To see $f$ is CC-regular, let $\left(z_{n}\right)$ be a cofinally Cauchy sequence in $(X, d)$. If there exists an infinite subset $N^{\prime}$ of $\mathbf{N}$ such that $z_{k} \notin \bigcup_{i \in \mathbf{N}} B\left(x_{i}, \frac{\delta}{4}\right) \forall k \in N^{\prime}$, then $\left(f\left(z_{n}\right)\right)$ is a cofinally Cauchy sequence as $f\left(z_{k}\right)=0 \forall k \in N^{\prime}$. If that is not the case, then there exists an infinite subset $N^{\prime}$ of $\mathbf{N}$ such that $\forall k \in N^{\prime}, z_{k} \in B\left(x_{m}, \frac{\delta}{4}\right)$ for some $m \in \mathbf{N}$, because otherwise for $\frac{\delta}{4}>0$, there would not be any infinte subset $N_{\frac{\delta}{4}}$ of $\mathbf{N}$ 
such that $d\left(z_{n}, z_{m}\right)<\frac{\delta}{4} \forall n, m \in N_{\frac{\delta}{4}}$, which contradicts the fact that $\left(z_{n}\right)$ is cofinally Cauchy. Thus for some $t \geq m, f\left(z_{k}\right) \in[0, t] \forall k \in N^{\prime}$. Enumerate the elemets of $N^{\prime}$ in increasing order and let $N^{\prime}=\left\{k_{1}, k_{2}, k_{3}, \ldots\right\}$. Since $[0, t]$ is totally bounded in $\mathbf{R}$, $\left(f\left(z_{k_{n}}\right)\right)$ is cofinally Cauchy. Thus $\left(f\left(z_{n}\right)\right)$ is cofinally Cauchy. Hence $f$ is CC-regular and uniformly locally Lipschitz but unbounded. We get a contradiction.

We know that a metric space $(X, d)$ is $\mathrm{UC}$ if and only if each sequence $\left(x_{n}\right)$ in $X$ satisfying $\lim _{n \rightarrow \infty} I\left(x_{n}\right)=0$ clusters, where $I(x)=d(x, X \backslash\{x\})$ measures the isolation of $x$ in the space $[3,24]$. Similarly, a metric space $(X, d)$ is cofinally complete if and only if each sequence $\left(x_{n}\right)$ in $X$ satisfying $\lim _{n \rightarrow \infty} \nu\left(x_{n}\right)=0$ clusters, where $\nu(x)=\sup \left\{\epsilon>0: \operatorname{cl}\left(B_{d}(x, \epsilon)\right)\right.$ is compact $\}$ if $x$ has a compact neighborhood, and $\nu(x)=0$ otherwise [6]. This geometric functional is called the local compactness functional on $X$. The set $\{x \in X: \nu(x)=0\}$ is the set of points of non-local compactness of $X$, which is denoted by $\operatorname{nlc}(X)$. Thus a metric space is said to be locally compact if $\nu(x)>0 \forall x \in X$, while it is called uniformly locally compact if $\inf \{\nu(x): x \in X\}>0$. Now we are ready to give a few more results.

We have already noted that every uniformly continuous function between two metric spaces is continuous and CC-regular. We now look for the conditions under which the converse is true.

Theorem 3.18. Let $(X, d)$ be a metric space. Then the following are equivalent:

(a) $(X, d)$ is a $U C$ space.

(b) Each continuous $C C$-regular function from $(X, d)$ with values in a metric space $(Y, \rho)$ is uniformly continuous.

(c) Each locally Lipschitz CC-regular function from $(X, d)$ with values in a metric space $(Y, \rho)$ is uniformly continuous.

(d) Each real-valued locally Lipschitz CC-regular function on $(X, d)$ is uniformly continuous.

(e) Each continuous $P C$-regular function from $(X, d)$ with values in a metric space $(Y, \rho)$ is uniformly continuous.

(f) Each locally Lipschitz $P C$-regular function from $(X, d)$ with values in a metric space $(Y, \rho)$ is uniformly continuous.

(g) Each real-valued locally Lipschitz PC-regular function on $(X, d)$ is uniformly continuous.

(h) Each continuous uniformly locally bounded function from $(X, d)$ with values in a metric space $(Y, \rho)$ is uniformly continuous.

(i) Each locally Lipschitz uniformly locally bounded function from $(X, d)$ with values in a metric space $(Y, \rho)$ is uniformly continuous.

(j) Each real-valued locally Lipschitz uniformly locally bounded function on $(X, d)$ is uniformly continuous.

Proof. The statements (a) $\Rightarrow(\mathrm{b}) \Rightarrow(\mathrm{c}) \Rightarrow(\mathrm{d}),(\mathrm{a}) \Rightarrow$ (e) $\Rightarrow$ (f) $\Rightarrow$ (g) and (a) $\Rightarrow(\mathrm{h}) \Rightarrow(\mathrm{i}) \Rightarrow(\mathrm{j})$ are all immediate.

$(\mathrm{d}) \Rightarrow(\mathrm{a})$ : First we prove that $(X, d)$ is complete. Suppose $\left(x_{n}\right)$ is a Cauchy sequence of distinct points in $(X, d)$ such that it does not converge. Thus the set $A=\left\{x_{n}: n \in \mathbf{N}\right\}$ is closed and discrete. Consequently, $\forall n \in \mathbf{N}, \exists \epsilon_{n}>0$ such that $d\left(x_{m}, x_{n}\right)>\epsilon_{n} \forall m \neq n$. Let $\delta_{n}=\min \left\{1 / n, \epsilon_{n} / 3\right\}$. Define a function $f:(X, d) \rightarrow$ $[0,2]$ as follows: 


$$
f(x)= \begin{cases}1-\frac{1}{\delta_{n}} d\left(x, x_{n}\right), & x \in B\left(x_{n}, \delta_{n}\right) \text { for some odd } n \in \mathbf{N}, \\ 2-\frac{2}{\delta_{n}} d\left(x, x_{n}\right), & x \in B\left(x_{n}, \delta_{n}\right) \text { for some even } n \in \mathbf{N}, \\ 0, & \text { otherwise. }\end{cases}
$$

Clearly, $f$ restricted to each ball $B\left(x_{n}, \delta_{n}\right)$ is Lipschitz. Let $x \in X$, since $x$ is not a cluster point of the sequence $\left(x_{n}\right)$ and $\epsilon_{n} \leq 1 / n \forall n \in \mathbf{N}, \exists \delta_{x}>0$ such that $B\left(x, \delta_{x}\right)$ intersects at most one of the balls $B\left(x_{n}, \delta_{n}\right)$. Thus $f$ is locally Lipschitz. Since the range of $f$ is totally bounded, $f$ is CC-regular as well, but $f$ is not uniformly continuous since every uniformly continuous functions is Cauchy-continuous. We get a contradiction. Hence $(X, d)$ is a complete metric space.

To see that $(X, d)$ is a UC space, let $\left(x_{n}\right)$ be a sequence of distinct points in $X$ such that $\lim _{n \rightarrow \infty} I\left(x_{n}\right)=0$. Suppose the sequence does not cluster. Since $(X, d)$ is complete, $\left(x_{n}\right)$ has no Cauchy subsequence. Thus $\exists \delta>0$ such that by passing to a subsequence, if needed, the family of open balls $\left\{B\left(x_{n}, \delta\right): n \in \mathbf{N}\right\}$ is pairwise disjoint. Also, $\lim _{n \rightarrow \infty} I\left(x_{n}\right)=0$ implies that we can assume $I\left(x_{n}\right)<\min \left\{\delta, \frac{1}{n}\right\} \forall n \in$ $\mathbf{N}$. Let $\delta_{n}=\min \left\{\delta, \frac{1}{n}\right\} \forall n \in \mathbf{N}$. Consequently, $\forall n \in \mathbf{N}$, we can choose $y_{n} \neq x_{n}$ such that $d\left(x_{n}, y_{n}\right)<\delta_{n}$. Let $\epsilon_{n}=d\left(x_{n}, y_{n}\right) \forall n \in \mathbf{N}$. Define a function $f:(X, d) \rightarrow[0,1]$ as follows:

$$
f(x)= \begin{cases}1-\frac{1}{\epsilon_{n}} d\left(x, x_{n}\right), & x \in B\left(x_{n}, \epsilon_{n}\right) \text { for some } n \in \mathbf{N}, \\ 0, & \text { otherwise. }\end{cases}
$$

Note that $f$ is locally Lipschitz and CC-regular but not uniformly continuous as $d\left(x_{n}, y_{n}\right)$ tends to 0 but $\left|f\left(x_{n}\right)-f\left(y_{n}\right)\right|=1 \forall n \in \mathbf{N}$. We get a contradiction. Thus $(X, d)$ is a $\mathrm{UC}$ space.

In a manner similar to the proof of $(\mathrm{d}) \Rightarrow(\mathrm{a})$, the implications $(\mathrm{g}) \Rightarrow$ (a) and (j) $\Rightarrow$ (a) can be proved.

Here we would like to mention that if we consider the function in Example 3.15 with the usual metric on the set $Y$, the function would be Cauchy-Lipschitz but not uniformly locally bounded. Thus a Cauchy-Lipschitz function between two metric spaces need not be uniformly locally bounded.

Since a UC space is complete, it is natural to consider the metric spaces whose completions are UC. In our next theorem, we present some new equivalent conditions for such metric spaces.

Theorem 3.19. Let $(X, d)$ be a metric space. Then the following are equivalent:

(a) The completion $(\widehat{X}, d)$ of $(X, d)$ is a UC space.

(b) Each Cauchy-continuous $C C$-regular function from $(X, d)$ with values in a metric space $(Y, \rho)$ is uniformly continuous.

(c) Each Cauchy-Lipschitz CC-regular function from $(X, d)$ with values in a metric space $(Y, \rho)$ is uniformly continuous.

(d) Each uniformly locally Lipschitz $C C$-regular function from $(X, d)$ with values in a metric space $(Y, \rho)$ is uniformly continuous.

(e) Each real-valued uniformly locally Lipschitz $C C$-regular function on $(X, d)$ is uniformly continuous.

(f) Each Cauchy-continuous $P C$-regular function from $(X, d)$ with values in a metric space $(Y, \rho)$ is uniformly continuous.

(g) Each Cauchy-Lipschitz PC-regular function from $(X, d)$ with values in a metric space $(Y, \rho)$ is uniformly continuous. 
(h) Each uniformly locally Lipschitz $P C$-regular function from $(X, d)$ with values in a metric space $(Y, \rho)$ is uniformly continuous.

(i) Each real-valued uniformly locally Lipschitz $P C$-regular function on $(X, d)$ is uniformly continuous.

(j) Each Cauchy-continuous uniformly locally bounded function from $(X, d)$ with values in a metric space $(Y, \rho)$ is uniformly continuous.

(k) Each Cauchy-Lipschitz uniformly locally bounded function from $(X, d)$ with values in a metric space $(Y, \rho)$ is uniformly continuous.

(l) Each real-valued Cauchy-Lipschitz uniformly locally bounded function on $(X, d)$ is uniformly continuous.

Proof. The implications (b) $\Rightarrow(\mathrm{c}) \Rightarrow(\mathrm{d}) \Rightarrow(\mathrm{e}),(\mathrm{f}) \Rightarrow(\mathrm{g}) \Rightarrow(\mathrm{h}) \Rightarrow(\mathrm{i}),(\mathrm{j}) \Rightarrow$ $(\mathrm{k}) \Rightarrow(\mathrm{l})$ are all immediate. The implications $(\mathrm{a}) \Rightarrow(\mathrm{b}),(\mathrm{a}) \Rightarrow(\mathrm{f})$ and $(\mathrm{a}) \Rightarrow(\mathrm{j})$ follow from the fact that the completion $(\widehat{X}, d)$ of a metric space $(X, d)$ is a UC space if and only if every Cauchy-continuous function $f:(X, d) \rightarrow(Y, \rho)$, where $(Y, \rho)$ is any metric space, is uniformly continuous [21].

(e) $\Rightarrow(\mathrm{a})$ : Suppose $(\widehat{X}, d)$ is not a UC space. Then there exists a sequence $\left(x_{n}\right)$ of distinct points with $\lim _{n \rightarrow \infty} I\left(x_{n}\right)=0$ such that $\left(x_{n}\right)$ does not have any Cauchy subsequence [21]. Thus $\exists \delta>0$ such that by passing to a subsequence, if needed, the family of open balls $\left\{B\left(x_{n}, \delta\right): n \in \mathbf{N}\right\}$ is pairwise disjoint. Let $\delta_{n}=$ $\min \left\{\frac{\delta}{4}, \frac{1}{n}\right\} \forall n \in \mathbf{N}$. Also, $\lim _{n \rightarrow \infty} I\left(x_{n}\right)=0$ implies that we can assume $I\left(x_{n}\right)<\delta_{n}$ $\forall n \in \mathbf{N}$. Consequently, $\forall n \in \mathbf{N}$, we can choose $y_{n} \neq x_{n}$ such that $d\left(x_{n}, y_{n}\right)<\delta_{n}$. Let $\epsilon_{n}=d\left(x_{n}, y_{n}\right) \forall n \in \mathbf{N}$. Define a function $f:(X, d) \rightarrow[0,1]$ as follows:

$$
f(x)= \begin{cases}1-\frac{1}{\epsilon_{n}} d\left(x, x_{n}\right), & x \in B\left(x_{n}, \epsilon_{n}\right) \text { for some } n \in \mathbf{N}, \\ 0, & \text { otherwise. }\end{cases}
$$

The function $f$ is uniformly locally Lipschitz because $\forall x \in X, B\left(x, \frac{\delta}{4}\right)$ intersects at most one of the balls $B\left(x_{m}, \frac{\delta}{4}\right)$ and $f$ restricted to each ball $B\left(x_{m}, \frac{\delta}{4}\right)$ is Lipschitz. Since $[0,1]$ is totally bounded in $\mathbf{R}, f$ is CC-regular as well. Thus $f$ is uniformly locally Lipschitz and CC-regular but not uniformly continuous, a contradiction.

(i) $\Rightarrow$ (a): In a manner similar to the proof of $(e) \Rightarrow(a),(i) \Rightarrow(a)$ can be proved.

$(\mathrm{l}) \Rightarrow(\mathrm{a})$ : Let $f:(\widehat{X}, d) \rightarrow \mathbf{R}$ be a locally Lipschitz uniformly locally bounded function. Since $(\widehat{X}, d)$ is a complete metric space, by Theorem 3.5 in [10], $f$ is CauchyLipschitz. This implies $\left.f\right|_{X}$ is Cauchy-Lipschitz and uniformly locally bounded and hence uniformly continuous. We claim that $f:(\widehat{X}, d) \rightarrow \mathbf{R}$ is uniformly continuous as well. For $\epsilon>0, \exists \delta>0$ such that $\forall x, y \in X$ with $d(x, y)<\delta,|f(x)-f(y)|<\epsilon / 3$. Let $\hat{x}, \hat{y} \in \widehat{X}$ such that $d(\hat{x}, \hat{y})<\delta / 4$. Since $\hat{x}, \hat{y} \in \widehat{X}$, there exists sequences $\left(x_{n}\right)$ and $\left(y_{n}\right)$ in $X$ such that $\left(x_{n}\right)$ converges to $\hat{x}$ and $\left(y_{n}\right)$ converges to $\hat{y}$. Thus, there exists $n_{0} \in \mathbf{N}$ such that $d\left(x_{n}, \hat{x}\right)<\delta / 2, d\left(y_{n}, \hat{y}\right)<\delta / 4,\left|f(\hat{x})-f\left(x_{n}\right)\right|<\epsilon / 3$ and $\left|f\left(y_{n}\right)-f(\hat{y})\right|<\epsilon / 3 \forall n \geq n_{0}$. Thus for all $n \geq n_{0}$,

$$
d\left(x_{n}, y_{n}\right) \leq d\left(x_{n}, \hat{x}\right)+d(\hat{x}, \hat{y})+d\left(y_{n}, \hat{y}\right)<\delta
$$

Therefore,

$$
|f(\hat{x})-f(\hat{y})| \leq\left|f(\hat{x})-f\left(x_{n_{0}}\right)\right|+\left|f\left(x_{n_{0}}\right)-f\left(y_{n_{0}}\right)\right|+\left|f\left(y_{n_{0}}\right)-f(\hat{y})\right|<\epsilon .
$$

Thus by Theorem $3.18,(\widehat{X}, d)$ is a UC space.

Now we study how the isolation functional $I$ and the local compactness functional $\nu$ are linked with continuous CC-regular functions. 
Theorem 3.20. Let $f$ be a function from a metric space $(X, d)$ to another metric space $(Y, \rho)$. If for some $\epsilon>0, f$ is uniformly continuous on $\{x \in X: I(x)<\epsilon\}$, then $f$ is continuous and $C C$-regular on $X$.

Proof. Let $\left(x_{n}\right)$ be a sequence of distinct points in $X$ converging to a point $x \in X$. Then there exists $n_{0} \in \mathbf{N}$ such that $d\left(x_{n}, x_{m}\right)<\frac{\epsilon}{2} \forall n, m \geq n_{0}$. Thus, $f$ is uniformly continuous on the set $\{x\} \cup\left\{x_{n}: n \geq n_{0}\right\}$. But this implies that $\left(f\left(x_{n}\right)\right)$ converges to $f(x)$. Hence the function is continuous.

Now suppose the function is not CC-regular. Therefore $\exists \epsilon_{o}>0$ such that $\forall n \in$ $\mathbf{N}, \exists x_{n} \in X$ such that $f\left(B\left(x_{n}, \frac{1}{n}\right)\right)$ cannot be contained in any finite union of open balls of radius $\epsilon_{o}$ in $Y$. Using the same technique as in Theorem 2.5, we can choose a sequence $\left(z_{n}\right)$ in $X$ consisting of infinite elements from each ball $B\left(x_{n}, \frac{1}{n}\right)$ such that the image of the sequence is $\frac{\epsilon_{o}}{2}$ discrete. Choose $\frac{1}{n_{o}}<\epsilon$. Since the function is uniformly continuous on the set $A=\{x \in X: I(x)<\epsilon\}, \exists \delta>0$ such that $\forall x, y \in A$ with $d(x, y)<\delta, \rho(f(x), f(y))<\frac{\epsilon_{o}}{2}$. Choose $\frac{2}{m}<\min \left\{\frac{1}{n_{o}}, \delta\right\}$. Let $\{y, z\} \subset B\left(x_{m}, \frac{1}{m}\right)$, where $y \neq z$ such that $\{y, z\} \subset\left\{z_{n}: n \in \mathbf{N}\right\}$. Now, $\{y, z\} \subset A$ with $d(y, z)<\delta$ but $\rho(f(y), f(z))>\frac{\epsilon_{o}}{2}$. We get a contradiction.

Remark 3.21. The converse of the above result may not be true. For example, the function $f$ defined in Remark 2.4 is continuous and CC-regular, but there does not exist any $\epsilon>0$ such that $f$ is uniformly continuous on $\{x \in X: I(x)<\epsilon\}$.

Also, if we replace the isolation functional with the local compactness functional in the hypothesis of the previous result, then $f$ need not be continuous. For example, take any discontinuous function from $\mathbf{R}$ to $\mathbf{R}$. For the local compactness functional, we have the following analogous result which can be proved in a manner similar to that of Theorem 3.20.

Theorem 3.22. Let $f$ be a continuous function from a metric space $(X, d)$ to another metric space $(Y, \rho)$. If for some $\epsilon>0, f$ is uniformly continuous on $\{x \in X: \nu(x)<\epsilon\}$, then $f$ is $C C$-regular on $X$.

Theorem 3.23. Let $(X, d)$ be a metric space. Suppose for every real-valued continuous function $f$ defined on it, there exists some $\lambda>0$ such that $f$ is uniformly continuous on $\{x \in X: \nu(x)<\lambda\}$. Then $(X, d)$ is cofinally complete.

Proof. Let $\left(x_{n}\right)$ be a cofinally Cauchy sequence of distinct points in $X$. By Proposition 2.4 in [6], there exists a pairwise disjoint family $\left\{\mathbf{M}_{j}: j \in \mathbf{N}\right\}$ of infinite subsets of $\mathbf{N}$ such that if $i \in \mathbf{M}_{j}$ and $l \in \mathbf{M}_{j}$ then $d\left(x_{i}, x_{l}\right)<\frac{1}{j}$. Suppose the sequence $\left(x_{n}\right)$ does not cluster, thus the set $A=\left\{x_{n}: n \in \mathbf{N}\right\}$ is closed and discrete. Enumerate the elements of $\mathbf{M}_{n}$ as $l_{1}^{n}, l_{2}^{n}, l_{3}^{n}, \ldots$ for each $n \in \mathbf{N}$. Therefore, for each $n$, there exists unique $i, k \in \mathbf{N}$ such that $n=l_{i}^{k}$. Define a function $f$ on $A$ such that $\forall k \in \mathbf{N}, f\left(x_{l_{i}^{k}}\right)=1$ if $i$ is even and $f\left(x_{l_{i}^{k}}\right)=2$ if $i$ is odd. Since $f$ is continuous and CC-regular on $A$, extend it to a function $F$ on $X$ such that $F$ is continuous and CC-regular.

We claim that there does not exist any $\lambda>0$ such that $F$ is uniformly continuous on $\{x \in X: \nu(x)<\lambda\}$. Suppose the claim is not true. Therefore $\exists \lambda>0$ such that $F$ is uniformly continuous on $\{x \in X: \nu(x)<\lambda\}$. Choose $\frac{1}{n_{o}}<\lambda$, consequently $\nu\left(x_{k}\right)<\frac{1}{n_{o}}<\lambda \forall x_{k} \in \bigcup_{n \geqslant n_{o}} \mathbf{M}_{n}$. But $F$ is not uniformly continuous on $\bigcup_{n \geqslant n_{o}} \mathbf{M}_{n}$. We arrive at a contradiction. 


\section{Cofinally Bourbaki-Cauchy regular functions vis-á-vis CC-regular functions}

In order to sequentially characterize the metric spaces on which every real-valued uniformly continuous function is bounded, recently in 2014 Garrido and Meroño [17] defined two new classes of sequences which they called Bourbaki-Cauchy and cofinally Bourbaki-Cauchy. Cofinally Bourbaki-Cauchy sequences are weaker than Bourbaki-Cauchy sequences as well as cofinally Cauchy sequences. In this section, we deal with cofinally Bourbaki-Cauchy regular functions and their relation with CC-regular functions. Let us first mention some relevant definitions.

Definitions 4.1. Let $(X, d)$ be a metric space and $\epsilon$ be a positive number, then an ordered set of points $\left\{x_{0}, x_{1}, \ldots, x_{m}\right\}$ in $X$ satisfying $d\left(x_{i-1}, x_{i}\right)<\epsilon$ for $i=$ $1,2, \ldots, m$, is said to be an $\epsilon$-chain of length $m$ from $x_{o}$ to $x_{m}$. We call $X \epsilon$-chainable if each two points in $X$ can be joined by an $\epsilon$-chain, and $X$ is called chainable if $X$ is $\epsilon$-chainable for every $\epsilon>0$. Let $A$ be a subset of $X$. Then, $A$ is said to be finitely chainable in $X$ if for every $\epsilon>0$, there are finitely many points $p_{1}, p_{2}, \ldots, p_{r}$ in $X$ and a positive integer $m$ such that every point of $A$ can be joined with some $p_{j}, 1 \leq j \leq r$ by an $\epsilon$-chain of length $m$.

These finitely chainable sets are also called Bourbaki-bounded sets in the literature, $[8,9,17]$, because these sets were considered in the book of Bourbaki [12]. These sets are simply called bounded sets in $[12,18]$, where these sets are considered in the frame of uniform spaces. Observe that every totally bounded subset of a metric space $(X, d)$ is finitely chainable in $(X, d)$. Also, a bounded subset of a metric space $(X, d)$ need not be finitely chainable in $(X, d)$, for example, consider any infinite subset of a metric space $(X, d)$, where $d$ is the zero-one metric.

Definitions 4.2. Let $(X, d)$ be a metric space. A sequence $\left(x_{n}\right)$ is said to be:

(a) Bourbaki-Cauchy in $X$ if for every $\epsilon>0$, there exist $m \in \mathbf{N}$ and $n_{o} \in \mathbf{N}$ such that whenever $n>j \geq n_{o}$, the points $x_{j}$ and $x_{n}$ can be joined by an $\epsilon$-chain of length $m$.

(b) cofinally Bourbaki-Cauchy in $X$ if for every $\epsilon>0$, there exist $m \in \mathbf{N}$ and an infinite subset $\mathbf{N}_{\epsilon}$ of $\mathbf{N}$ such that the points $x_{j}$ and $x_{n}$ can be joined by an $\epsilon$-chain of length $m$ for every $j, n \in \mathbf{N}_{\epsilon}$.

Moreover, a metric space $(X, d)$ is said to be (cofinally) Bourbaki-complete if every (cofinally) Bourbaki-Cauchy sequence in $(X, d)$ clusters.

Definitions 4.3. A function $f$ from a metric space $(X, d)$ to another metric space $(Y, \rho)$ is said to be Bourbaki-Cauchy regular (or BC-regular for short) if $\left(f\left(x_{n}\right)\right)$ is Bourbaki-Cauchy in $(Y, \rho)$ for every Bourbaki-Cauchy sequence $\left(x_{n}\right)$ in $(X, d)$. A function $f$ from a metric space $(X, d)$ to another metric space $(Y, \rho)$ is said to be cofinally Bourbaki-Cauchy regular (or CBC-regular for short) if $\left(f\left(x_{n}\right)\right)$ is cofinally Bourbaki-Cauchy in $(Y, \rho)$ for every cofinally Bourbaki-Cauchy sequence $\left(x_{n}\right)$ in $(X, d)$.

First we study an interesting characterization of CBC-regular functions which is similar to a characterization of CC-regular functions (see Theorem 2.8). Note that for a subset $A$ of a metric space $(X, d)$ and $\epsilon>0, A^{\epsilon}=\bigcup\{B(x, \epsilon): x \in A\}$, $B^{1}(x, \epsilon)=B(x, \epsilon)$ and for every $n \geq 2, B^{n}(x, \epsilon)=\left(B^{n-1}(x, \epsilon)\right)^{\epsilon}$.

Theorem 4.4. Let $f$ be a function from a metric space $(X, d)$ to another metric space $(Y, \rho)$. The following statements are equivalent: 
(a) $f$ is $C B C$-regular.

(b) $\forall \epsilon>0, \exists \delta>0$ such that $\forall x \in X$ and $m \in \mathbf{N}, \exists n \in \mathbf{N}$ and $\left\{y_{1}, y_{2}, \ldots y_{k}\right\} \subseteq$ $Y$ such that $f\left(B^{m}(x, \delta)\right) \subseteq \bigcup_{i=1}^{k} B^{n}\left(y_{i}, \epsilon\right)$.

Proof. (a) $\Rightarrow$ (b): Suppose $\exists \epsilon>0$ such that $\forall \delta>0, \exists x_{\delta}$ and $m_{x}$ such that $f\left(B^{m_{x}}\left(x_{\delta}, \delta\right)\right)$ cannot be bounded by any finite union of $n^{t h} \epsilon$ - enlarged open balls. For each $n \in \mathbf{N}$, let $\delta_{n}=\frac{1}{n}$, thus there exists $x_{n}$ and $m_{x_{n}}$ (say $m_{n}$ ) such that $f\left(B^{m_{n}}\left(x_{n}, \frac{1}{n}\right)\right)$ cannot be contained in any finite union of the type $\bigcup_{i=1}^{k} B^{l}\left(y_{i}, \epsilon\right)$ for all $k, l \in \mathbf{N}$. Let $a_{1}^{n} \in f\left(B^{m_{n}}\left(x_{n}, \frac{1}{n}\right)\right)$. Since $f\left(B^{m_{n}}\left(x_{n}, \frac{1}{n}\right)\right) \subsetneq B\left(a_{1}^{n}, \epsilon\right)$, there exists $a_{2}^{n} \in f\left(B^{m_{n}}\left(x_{n}, \frac{1}{n}\right)\right)$ such that $a_{2}^{n} \notin B\left(a_{1}^{n}, \epsilon\right)$. Similarly, there exists $a_{3}^{n} \in f\left(B^{m_{n}}\left(x_{n}, \frac{1}{n}\right)\right)$ such that $a_{3}^{n} \notin B^{2}\left(a_{1}^{n}, \epsilon\right) \cup B^{2}\left(a_{2}^{n}, \epsilon\right)$. Thus we get a sequence $\left(a_{m}^{n}\right)_{m \in \mathbf{N}} \subseteq f\left(B^{m_{n}}\left(x_{n}, \frac{1}{n}\right)\right)$ such that $a_{j}^{n}$ cannot be bound with $a_{i}^{n}$ by an $\epsilon$-chain of length $j-1$ for $0<i<j$. Let $A_{n}=\left\{a_{m}^{n}: m \in \mathbf{N}\right\}$. Now we will construct a sequence in $Y$. Let $F_{1}=\left\{a_{\alpha_{1}}^{1}\right\}$ where $\alpha_{1} \geqslant 1$. Let $a_{\alpha_{2}}^{2} \in A_{2}\left(\alpha_{2} \geqslant 2\right)$ such that $a_{\alpha_{2}}^{2}$ cannot be joined with $a_{\alpha_{1}}^{1}$ by an $\epsilon$-chain of length 1 . It is possible because if there does not exist such $a_{\alpha_{2}}^{2}$, then all the elements $\left(a_{m}^{n}\right)_{m \geqslant \max \left\{\alpha_{1}, 2\right\}}$ can be joined by an $\epsilon$-chain of length 2 , but it is a contradiction. Similarly, choose $a_{\alpha_{3}}^{1} \in A_{1}\left(\alpha_{3} \geqslant 3\right)$ such that $a_{\alpha_{3}}^{1}$ cannot be joined by an $\epsilon$ - chain of length 2 with the elements $a_{\alpha_{1}}^{1}$ and $a_{\alpha_{2}}^{2}$. Let $F_{2}=\left\{a_{\alpha_{1}}^{1}, a_{\alpha_{2}}^{2}, a_{\alpha_{3}}^{1}\right\}$. Now suppose a finite subset $F_{n}=\left\{a_{\alpha_{1}}^{1}, a_{\alpha_{2}}^{2}, a_{\alpha_{3}}^{1}, \ldots a_{\alpha_{\frac{n(n+1)}{2}}}^{1}\right\}$ of $\bigcup_{i=1}^{n} A_{i}$ is chosen such that $a_{\alpha_{j}}^{l}$ cannot be bound with $a_{\alpha_{i}}^{m}$ by an $\epsilon$-chain of length $j-1$ for all $0<i<j(1 \leqslant l, m \leqslant n)$ and $\left|F_{n} \cap A_{i}\right|=n-i+1$ for all $1 \leq i \leq n$.

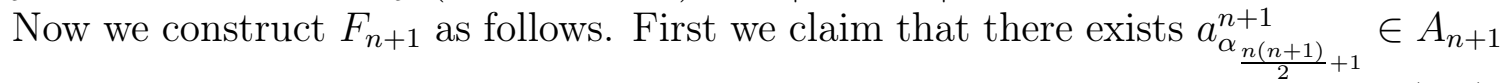
such that it cannot be joined with any member of $F_{n}$ by an $\epsilon$-chain of length $\frac{n(n+1)}{2}$. Let $k \geqslant \max \left\{\alpha_{\underline{n(n+1)}}, \frac{n(n+1)}{2}+1\right\}$. Consider $\left\{a_{m}: m \geq 2 k\right\}$. Since $\left\{a_{m}: m \geq 2 k\right\}$ is infinite and $F_{n}$ is finite, there exists $y, y^{\prime} \in\left\{a_{m}: m \geqslant 2 k\right\} \backslash F_{n}, y \neq y^{\prime}$, such that $y$ and $z$ can be joined by an $\epsilon$-chain of length $\frac{n(n+1)}{2}$ and $y^{\prime}$ and $z$ can also be joined by an $\epsilon$-chain of length $\frac{n(n+1)}{2}$. Thus $y$ and $y^{\prime}$ can be joined by an $\epsilon$-chain of length $n(n+1)$. We get a contradiction. Repeating this process for $i=n, n-1, \ldots, 1$ and for the set $F_{n} \cup\left\{a_{\frac{\alpha_{n(n+1)}}{n+1}+1}^{n+1}, a_{\frac{\alpha_{n(n+1)}}{2}+2}^{n}, \ldots, a_{\frac{\alpha_{\frac{n(n+1)}{2}}}{i}+(n-i+2)}\right\}$ together with the infinite set $E_{i}$, we get the desired set $F_{n+1}$. Now, if we choose a sequence in the order we picked the elements, that is, $a_{\alpha_{1}}^{1}, a_{\alpha_{2}}^{2}, a_{\alpha_{3}}^{1}, a_{\alpha_{4}}^{3}, \ldots$, then this sequence is not cofinally Bourbaki-Cauchy, but its preimage is cofinally Bourbaki-Cauchy. We get a contradiction.

(b) $\Rightarrow\left(\right.$ a): Let $\left(x_{n}\right)$ be a cofinally Bourbaki-Cauchy sequence. We claim that $\left(f\left(x_{n}\right)\right)$ is cofinally Bourbaki-Cauchy. Let $\epsilon>0$. Thus $\exists \delta>0$ such that $\forall x \in X$ and $m \in \mathbf{N}, \exists n \in \mathbf{N}$ and $\left\{y_{1}, y_{2}, \ldots y_{k}\right\} \subseteq Y$ such that $f\left(B^{m}(x, \delta)\right) \subseteq \bigcup_{i=1}^{k} B^{n}\left(y_{i}, \epsilon\right)$. Since $\left(x_{n}\right)$ is cofinally Bourbaki-Cauchy, there exist $m \in \mathbf{N}$ and an infinite subset $\mathbf{N}_{\delta}$ of $\mathbf{N}$ such that the points $x_{j}$ and $x_{n}$ can be joined by an $\delta$-chain of length $m$ for every $j, n \in \mathbf{N}_{\delta}$. Let $t \in \mathbf{N}_{\delta}$ and consider $B^{m}\left(x_{t}, \delta\right)$. Therefore, $\exists n \in \mathbf{N}$ and $\left\{y_{1}, y_{2}, \ldots y_{k}\right\} \subseteq Y$ such that $f\left(B^{m}\left(x_{t}, \delta\right)\right) \subseteq \bigcup_{i=1}^{k} B^{n}\left(y_{i}, \epsilon\right)$. Atleast one of these $B^{n}\left(y_{i}, \epsilon\right)$ will contain elements of the type $f\left(x_{k}\right)$ for infinite values of $k$. Hence $\left(f\left(x_{n}\right)\right)$ is cofinally Bourbaki-Cauchy.

Now we would like to discuss a sequential characterization of CBC-regular functions for which the following definitions are required. 
Definitions 4.5. A pair of sequences $\left(x_{n}\right)$ and $\left(y_{n}\right)$ in a metric space $(X, d)$ is said to be:

(a) Bourbaki asymptotic, written $\left(x_{n}\right) \asymp_{b}\left(y_{n}\right)$, if $\forall \epsilon>0, \exists m, n_{o} \in \mathbf{N}$ such that $x_{n}$ and $y_{n}$ can be joined by an $\epsilon$-chain of length $m \forall n \geq n_{o}$.

(b) cofinally Bourbaki asymptotic, written $\left(x_{n}\right) \asymp_{c b}\left(y_{n}\right)$, if $\forall \epsilon>0, \exists m \in \mathbf{N}$ and an infinite subset $N_{\epsilon}$ of $\mathbf{N}$ such that $x_{n}$ and $y_{n}$ can be joined by an $\epsilon$-chain of length $m \forall n \in N_{\epsilon}$.

(c) cofinally Bourbaki uniformly asymptotic, written $\left(x_{n}\right) \asymp_{c b}^{u}\left(y_{n}\right)$, if $\forall \epsilon>0, \exists m \in$ $\mathbf{N}$ and an infinite subset $N_{\epsilon}$ of $\mathbf{N}$ such that $x_{n}$ and $y_{k}$ can be joined by an $\epsilon$-chain of length $m \forall n, k \in N_{\epsilon}$.

As an immediate consequence of these definitions, we have the following proposition, whose routine proof is omitted.

Proposition 4.6. Let $\left(x_{n}\right)$ and $\left(y_{n}\right)$ be sequences in a metric space $(X, d)$.

(a) $\left(x_{n}\right)$ is cofinally Bourbaki-Cauchy in $(X, d)$ if and only if $\left(x_{n}\right) \asymp_{c b}^{u}\left(x_{n}\right)$.

(b) If $\left(x_{n}\right) \asymp_{c b}^{u}\left(y_{n}\right)$, then $\left(x_{n}\right)$ and $\left(y_{n}\right)$ are cofinally Bourbaki-Cauchy sequences.

Now we state another characterization of CBC-regular functions, which can be proved by using the above proposition.

Proposition 4.7. Let $f:(X, d) \rightarrow(Y, \rho)$ be a function between two metric spaces. The following statements are equivalent:

(a) $f$ is $C B C$-regular.

(b) If $\left(x_{n}\right)$ is a cofinally Bourbaki-Cauchy sequence in $(X, d)$ and $\left(f\left(x_{n}\right)\right) \asymp_{b}$ $\left(f\left(z_{n}\right)\right)$, where $\left(z_{n}\right)$ is any sequence in $X$, then $\left(f\left(x_{n}\right)\right) \asymp_{c b}^{u}\left(f\left(z_{n}\right)\right)$.

(c) If $\left(x_{n}\right) \asymp_{c b}^{u}\left(z_{n}\right)$ and $\left(f\left(x_{n}\right)\right) \asymp_{b}\left(f\left(z_{n}\right)\right)$, then $\left(f\left(x_{n}\right)\right) \asymp_{c b}^{u}\left(f\left(z_{n}\right)\right)$.

Theorem 4.8. Let $(X, d)$ be a metric space. Then the following statements are equivalent:

(a) $(X, d)$ is cofinally Bourbaki-complete.

(b) Whenever $f:(X, d) \rightarrow \mathbf{R}$ is a continuous $C B C$-regular function such that $f$ is never zero, then $\frac{1}{f}$ is also continuous and $C B C$-regular.

Proof. $\quad(\mathrm{a}) \Rightarrow(\mathrm{b})$ : Since every real-valued continuous function on a cofinally Bourbaki-complete metric space is CBC- regular [2], the statement holds.

(b) $\Rightarrow(\mathrm{a})$ : If $(X, d)$ is not cofinally Bourbaki-complete, then there exists a cofinally Bourabki-Cauchy sequence $\left(x_{n}\right)$ of distinct points in $(X, d)$ with no cluster point. Thus, $A=\left\{x_{n}: n \in \mathbf{N}\right\}$ is closed and discrete. Define a function $f$ from $A$ to $\mathbf{R}$ such that $f\left(x_{n}\right)=\frac{1}{n} \forall n \in \mathbf{N}$. Clearly, $f$ is continuous and CBC-regular. By Theorem 5.1 in [15, p. 149], there exists a continuous function $F: X \rightarrow(0,2)$ which extends $f$. Now $(0,2)$ is finitely chainable in $\mathbf{R}$ and since every sequence in a finitely chainable metric space is cofinally Bourbaki-Cauchy [17], $F$ is CBC-regular. Now, $F$ is never zero but $\frac{1}{F}$ is not CBC-regular, as $\left(x_{n}\right)$ is cofinally Bourbaki-Cauchy but $(n)$ is not cofinally Bourbaki-Cauchy in $\mathbf{R}$.

It is evident that cofinally Bourbaki-complete metric spaces are stronger than Bourbaki-complete metric spaces as well as cofinally complete metric spaces. In [2], it has been shown that neither a CC-regular function between two metric spaces need to be CBC-regular nor a CBC-regular function between two metric spaces need to be CC-regular. Similar observation has also been given for BC-regular and CBC-regular functions. In our next results, we establish some relations between the aforesaid functions. In the process, we find some equivalent conditions under which cofinally 
complete metric spaces and Bourbaki-complete metric space are cofinally Bourbakicomplete.

Theorem 4.9. Let $(X, d)$ be a metric space. Then the following statements are equivalent:

(a) $(X, d)$ is cofinally Bourbaki-complete.

(b) $(X, d)$ is cofinally complete and every $C C$-regular function from $(X, d)$ to any other metric space $(Y, \rho)$ is $C B C$-regular.

(c) $(X, d)$ is cofinally complete and every real-valued CC-regular function on $(X, d)$ is $C B C$-regular.

(d) $(X, d)$ is cofinally complete and every cofinally Bourbaki-Cauchy sequence in $(X, d)$ is cofinally Cauchy.

Proof. The implications $(\mathrm{b}) \Rightarrow(\mathrm{c})$ and $(\mathrm{d}) \Rightarrow(\mathrm{a})$ are immediate.

$(\mathrm{a}) \Rightarrow(\mathrm{b})$ : Let $f:(X, d) \rightarrow(Y, \rho)$ be a CC-regular function. Let $\left(x_{n}\right)$ be a cofinally Bourbaki-Cauchy sequence. Since $(X, d)$ is cofinally Bourbaki-complete, there exists a convergent subsequence $\left(x_{n_{k}}\right)$ of $\left(x_{n}\right)$. This implies $\left(x_{n_{k}}\right)$ is cofinally Cauchy and so is $\left(f\left(x_{n_{k}}\right)\right)$. Thus, $\left(f\left(x_{n}\right)\right)$ is cofinally Bourbaki-Cauchy, which implies $f$ is CBC-regular.

$(c) \Rightarrow(d)$ : Suppose there exists a cofinally Bourbaki-Cauchy sequence $\left(x_{n}\right)$ of distinct points in $(X, d)$ such that it is not cofinally Cauchy. Now, define a function $f: X \rightarrow \mathbf{R}$ as

$$
f(x)= \begin{cases}n, & x=x_{n} \text { for some } n \in \mathbf{N}, \\ 0, & \text { otherwise. }\end{cases}
$$

Clearly, $f$ is CC-regular but not CBC-regular, a contradiction.

Theorem 4.10. Let $(X, d)$ be a metric space. Then the following statements are equivalent:

(a) $(\widehat{X}, d)$ is cofinally Bourbaki-complete.

(b) $(\widehat{X}, d)$ is cofinally complete and every $C C$-regular function from $(X, d)$ to any other metric space $(Y, \rho)$ is $C B C$-regular.

(c) $(\widehat{X}, d)$ is cofinally complete and every real-valued CC-regular function on $(X, d)$ is $C B C$-regular.

(d) $(\widehat{X}, d)$ is cofinally complete and every cofinally Bourbaki-Cauchy sequence in $(X, d)$ is cofinally Cauchy.

Proof. $(\mathrm{a}) \Rightarrow(\mathrm{b})$ : Since $(\widehat{X}, d)$ is cofinally Bourbaki-complete, every cofinally Bourbaki-sequence in it has a Cauchy subsequence and hence is cofinally Cauchy.

(b) $\Rightarrow(c)$ : This is immediate.

$(c) \Rightarrow(d)$ : This can be proved in a manner similar to the proof of $(c) \Rightarrow(d)$ in Theorem 4.9 .

$(\mathrm{d}) \Rightarrow(\mathrm{a})$ : This follows from the fact that $(\widehat{X}, d)$ is cofinally Bourbaki-complete if and only if every cofinally Bourbaki-Cauchy sequence in it has a Cauchy subsequence.

Remark 4.11. Note that if a metric space $(X, d)$ or its completion $(\widehat{X}, d)$ is cofinally Bourbaki-complete, then every cofinally Bourbaki-Cauchy sequence in $(X, d)$ is cofinally Cauchy. But the converse need not hold. For example, as a metric subspace of the Hilbert space $l_{2}$, consider $X=\left\{e_{n}+\frac{1}{n} e_{k}: n, k \in \mathbf{N}\right\}$. Then $(X, d)$ is complete 
and every cofinally Bourbaki-Cauchy sequence in $(X, d)$ is cofinally Cauchy (the metric $d$ is induced by the $l_{2}$-norm), but $(X, d)$ is not cofinally Bourbaki-complete. Thus the conditions that $(X, d)$ is cofinally complete and $(\widehat{X}, d)$ is cofinally complete in Theorem 4.9 and Theorem 4.10 respectively, cannot be dropped.

In a manner similar to the proof of Theorem 4.9, we can prove the following result.

Theorem 4.12. Let $(X, d)$ be a metric space. Then the following statements are equivalent:

(a) $(X, d)$ is cofinally Bourbaki-complete.

(b) $(X, d)$ is Bourbaki-complete and every $B C$-regular function from $(X, d)$ to any other metric space $(Y, \rho)$ is $C B C$-regular.

(c) $(X, d)$ is Bourbaki-complete and every real-valued $B C$-regular function on $(X, d)$ is $C B C$-regular.

(d) $(X, d)$ is Bourbaki-complete and every cofinally Bourbaki-Cauchy sequence in $(X, d)$ has a Bourbaki-Cauchy subsequence.

In a manner similar to the proof of Theorem 4.10, we can prove the following result.

Theorem 4.13. Let $(X, d)$ be a metric space. Then the following statements are equivalent:

(a) $(\widehat{X}, d)$ is cofinally Bourbaki-complete.

(b) $(\widehat{X}, d)$ is Bourbaki-complete and every $B C$-regular function from $(X, d)$ to any other metric space $(Y, \rho)$ is $C B C$-regular.

(c) $(\widehat{X}, d)$ is Bourbaki-complete and every real-valued $B C$-regular function on $(X, d)$ is $C B C$-regular.

(d) $(\widehat{X}, d)$ is Bourbaki-complete and every cofinally Bourbaki-Cauchy sequence in $(X, d)$ has a Bourbaki-Cauchy subsequence.

Remark 4.14. Note that if a metric space $(X, d)$ or its completion $(\widehat{X}, d)$ is cofinally Bourbaki-complete, then every cofinally Bourbaki-Cauchy sequence in $(X, d)$ has a Bourbaki-Cauchy subsequence. But the converse need not hold. For example, consider the real Hilbert space $l_{2}$. Let $X \subseteq l_{2}$ be defined as $X=C(0,2)$ (closed ball around 0 of radius 2$)$. The metric space $(X, d)$ is finitely chainable. Thus every cofinally Bourbaki-Cauchy sequence in it has a Bourbaki-Cauchy subsequence (every sequence in a finitely chainable metric space has a Bourbaki-Cauchy subsequence [17]). $(X, d)$ is complete but not cofinally Bourbaki-complete because if we enumerate the elements of $A=\left\{e_{n}+\frac{1}{n} e_{k}: n, k \in \mathbf{N}\right\} \subset X$, we will get a cofinally Bourbaki-Cauchy sequence which does not have any cluster point in $X$. Thus the conditions that $(X, d)$ is Bourbaki-complete and $(\widehat{X}, d)$ is Bourbaki-complete in Theorem 4.12 and Theorem 4.13 respectively cannot be dropped.

In 1958, Atsuji proved: every real-valued uniformly continuous function on a metric space $(X, d)$ is bounded if and only if $(X, d)$ is finitely chainable [3]. It is known that the finite chainability of $(X, d)$ is also characterized by the boundedness of each Lipschitz in the small function on the space. This is implicit in Atsuji's proof and very explicit in [8]. In our next result, we study the boundedness of some combinations of Lipschitz-type functions with $\mathrm{CBC}$-regular functions. The metric spaces on which every real-valued continuous CBC-regular function is bounded are equivalent to finitely chainable metric spaces. 
Theorem 4.15. Let $(X, d)$ be a metric space. Then the following are equivalent:

(a) $(X, d)$ is finitely chainable.

(b) Whenever $(Y, \rho)$ is a metric space and $f:(X, d) \rightarrow(Y, \rho)$ is continuous and $C B C$-regular, then $f$ is bounded.

(c) Whenever $(Y, \rho)$ is a metric space and $f:(X, d) \rightarrow(Y, \rho)$ is locally Lipschitz and $C B C$-regular, then $f$ is bounded.

(d) Whenever $(Y, \rho)$ is a metric space and $f:(X, d) \rightarrow(Y, \rho)$ is Cauchy-Lipschitz and $C B C$-regular, then $f$ is bounded.

(e) Whenever $(Y, \rho)$ is a metric space and $f:(X, d) \rightarrow(Y, \rho)$ is uniformly locally Lipschitz and CBC-regular, then $f$ is bounded.

(f) Whenever $f$ is a real-valued uniformly locally Lipschitz and CBC-regular function on $(X, d)$, then $f$ is bounded.

Proof. The implications (b) $\Rightarrow(\mathrm{c}) \Rightarrow(\mathrm{d}) \Rightarrow(\mathrm{e}) \Rightarrow$ (f) are all immediate.

$(\mathrm{a}) \Rightarrow(\mathrm{b})$ : Let $f$ be a continuous CBC-regular function from $(X, d)$ to $(Y, \rho)$. Since a metric space $(X, d)$ is finitely chainable if and only if every sequence in it is cofinally Bourbaki-Cauchy [17], $f(X)$ is a finitely chainable subset of $(Y, \rho)$.

$(\mathrm{f}) \Rightarrow(\mathrm{a})$ : Suppose $(X, d)$ is not finitely chainable. Therefore, there exists $\epsilon>0$ such that for any finite subset $\left\{x_{1}, x_{2}, \ldots x_{n}\right\}$ of $X$ and for every $m \in \mathbf{N}$, there exists $x \in X$ such that $x$ cannot be joined with any $x_{i}$ by an $\epsilon$-chain of length $m$. Consider $a_{1} \in X$. Then for $m=1$, there exists $a_{2} \in X$ such that $a_{2}$ cannot be bound with $a_{1}$ by an $\epsilon$-chain of length 1 . By induction there exists $\left\{a_{n}: n \in \mathbf{N}\right\}$ where $a_{j}$ cannot be bound with $a_{i}$ by an $\epsilon$-chain of length $j-1$, for $0<i<j$. Define a function $f:(X, d) \rightarrow \mathbf{R}$ as follows:

$$
f(x)= \begin{cases}n-\frac{4 n}{\epsilon} d\left(x, a_{n}\right), & x \in B\left(a_{n}, \frac{\epsilon}{4}\right) \text { for some } n \in \mathbf{N}, \\ 0, & \text { otherwise. }\end{cases}
$$

Then, as in Theorem 3.17, the function $f$ is uniformly locally Lipschitz. To see $f$ is CBC-regular, let $\left(z_{n}\right)$ be a cofinally Bourbaki-Cauchy sequence in $(X, d)$. If there exists an infinite subset $N^{\prime}$ of $\mathbf{N}$ such that $z_{k} \notin \bigcup_{i \in \mathbf{N}} B\left(a_{i}, \frac{\epsilon}{4}\right) \forall k \in N^{\prime}$, then $\left(f\left(z_{n}\right)\right)$ is a cofinally Bourbaki-Cauchy sequence as $f\left(z_{k}\right)=0 \forall k \in N^{\prime}$. If that is not the case, then there exists an infinite subset $N^{\prime}$ of $\mathbf{N}$ such that $\forall k \in N^{\prime}, z_{k} \in B\left(a_{l}, \frac{\epsilon}{4}\right)$ for some $l \in \mathbf{N}$, because otherwise for $\frac{\epsilon}{4}>0$, there would not be any infinte subset $N_{\frac{\epsilon}{4}}$ of $\mathbf{N}$ and $m \in \mathbf{N}$ such that the points $z_{j}$ and $z_{n}$ can be joined by an $\frac{\epsilon}{4}$-chain of length $m$ for every $j, n \in \mathbf{N}_{\frac{\epsilon}{4}}$, which contradicts the fact that $\left(z_{n}\right)$ is cofinally BourbakiCauchy. Thus for some $t \geq l, f\left(z_{k}\right) \in[0, t] \forall k \in N^{\prime}$. Enumerate the elemets of $N^{\prime}$ in increasing order and let $N^{\prime}=\left\{k_{1}, k_{2}, k_{3}, \ldots\right\}$. Since $[0, t]$ is finitely chainable in $\mathbf{R}$, $\left(f\left(z_{k_{n}}\right)\right)$ is cofinally Bourbaki-Cauchy. Thus $\left(f\left(z_{n}\right)\right)$ is cofinally Bourbaki-Cauchy. Hence $f$ is CBC-regular and uniformly locally Lipschitz but unbounded. We get a contradiction.

Acknowledgements. The authors are thankful to Manisha Aggarwal for her various useful comments on many versions of this paper. The authors are also grateful to the Editor and the esteemed referee for his/her valuable suggestions.

\section{References}

[1] Aggarwal, M., and S. Kundu: More about the cofinally complete spaces and the Atsuji spaces. - Houston J. Math. 42:4, 2016, 1373-1395.

[2] Aggarwal, M., and S. Kundu: Boundedness of the relatives of uniformly continuous functions. - Topology Proc. 49, 2017, 105-119. 
[3] Atsuji, M.: Uniform continuity of continuous functions of metric spaces. - Pacific J. Math. 8, 1958, 11-16.

[4] BeER, G.: Metric spaces on which continuous functions are uniformly continuous and Hausdorff distance. - Proc. Amer. Math. Soc. 95:4, 1985, 653-658.

[5] BeER, G.: More about metric spaces on which continuous functions are uniformly continuous. - Bull. Aust. Math. Soc. 33:3, 1986, 397-406.

[6] Beer, G.: Between compactness and completeness. - Topology Appl. 155, 2008, 503-514.

[7] Beer, G., and G. Di Maio: The bornology of cofinally complete subsets. - Acta Math. Hungar. 134:3, 2012, 322-343.

[8] Beer, G., and M. I. Garrido: Bornologies and locally Lipschitz functions. - Bull. Aust. Math. Soc. 90, 2014, 257-263.

[9] Beer, G., and M. I. Garrido: Locally Lipschitz functions, cofinal completeness, and UC spaces. - J. Math. Anal. Appl. 428:2, 2015, 804-816.

[10] BeER, G., and M. I. GarRido: On the uniform approximation of Cauchy continuous functions. - Topology Appl. 208, 2016, 1-9.

[11] Beer, G., M. I. Garrido, and Ana S. Meroño: Uniform continuity and a new bornology for a metric space. - Set-Valued Var. Anal. 26:1, 2018, 49-65.

[12] Bourbaki, N.: Elements of mathematics, general topology, part 1. - Hermann, Paris, 1966.

[13] Bouziad, A., and E. Sukhacheva: Preservation of uniform continuity under pointwise product. - Topology Appl. 254, 2019, 132-144.

[14] Doss, R.: On uniformly continuous functions in metrizable spaces. - Proc. Math. Phys. Soc. Egypt 3, 1947, 1-6.

[15] Dugundji, J.: Topology. - Allyn and Bacon, Boston, 1966.

[16] Garrido, M. I., and J. A. Jaramillo: Lipschitz-type functions on metric spaces. - J. Math. Anal. Appl. 340:1, 2008, 282-290.

[17] Garrido, M. I., and A.S. Meroño: New types of completeness in metric spaces. - Ann. Acad. Sci. Fenn. Math. 39, 2014, 733-758.

[18] Hejcman, J.: Boundedness in uniform spaces and topological groups. - Czechoslovak Math. J. 9:84, 1959, 544-563.

[19] Howes, N. R.: On completeness. - Pacific J. Math. 38, 1971, 431-440.

[20] Howes, N. R.: Modern analysis and topology. - Springer-Verlag, New York, 1995.

[21] JAIn, T., and S. Kundu: Atsuji completions: Equivalent characterisations. - Topology Appl. $154: 1,2007,28-38$.

[22] Keremedis, K.: Metric spaces on which continuous functions are almost uniformly continuous. - Topology Appl. 232, 2017, 256-266.

[23] Kundu, S., M. Aggarwal, and S. Hazra: Finitely chainable and totally bounded metric spaces: Equivalent characterizations. - Topology Appl. 216, 2017, 59-73.

[24] Kundu, S., and T. Jain: Atsuji spaces: Equivalent conditions. - Topology Proc. 30:1, 2006, $301-325$.

[25] Nagata, J.: On the uniform topology of bicompactifications. - J. Inst. Polytech. Osaka City Univ. Ser. A. Math. 1, 1950, 28-38.

[26] Rice, M. D.: A note on uniform paracompactness. - Proc. Amer. Math. Soc. 62:2, 1977, $359-362$.

[27] Sмiтh, J.: Review of "A note on uniform paracompactness" by Michael D. Rice. - Math. Rev. $55,1978$.

[28] Snipes, R. F.: Functions that preserve Cauchy sequences. - Nieuw Arch. Wisk. 25:3, 1977, $409-422$. 
[29] Snipes, R. F.: Cauchy-regular functions. - J. Math. Anal. Appl. 79:1, 1981, 18-25.

[30] Toader, G.: On a problem of Nagata. - Mathematica 20:43(1), 1978, 77-79.

Received 28 January $2019 \bullet$ Accepted 10 September 2019 